Pacific

Journal of

Mathematics

\title{
A LOCAL-GLOBAL PROBLEM FOR LINEAR DIFFERENTIAL EQUATIONS
}

MARIUS VAN DER PUt AND MARC REVERSAT

Volume $238 \quad$ No. 1

November 2008 


\title{
A LOCAL-GLOBAL PROBLEM FOR LINEAR DIFFERENTIAL EQUATIONS
}

\author{
MARIUS VAN DER PUT AND MARC REVERSAT
}

\begin{abstract}
An inhomogeneous linear differential equation $L y=f$ over a global differential field can have a formal solution for each place without having a global solution. The vector space $\lg l(L)$ measures this phenomenon. This space is interpreted in terms of cohomology of linear algebraic groups and is computed for abelian differential equations and for regular singular equations. An analogue of Artin reciprocity for abelian differential equations is given. Malgrange's work on irregularity is reproved in terms cohomology of linear algebraic groups.
\end{abstract}

\section{Introduction}

The following topics have many common features: elliptic curves $E$ over a number field $K$; Drinfeld modules over a field $K$ like $\mathbb{F}_{q}(t)$; and linear differential equations over a differential field $K$, for example, a finite extension of the differential field $\mathbb{C}(z)$.

For every place $v$ of $K$ one considers the completion $K_{v}$. An example of a localglobal problem is the following. Consider an elliptic curve $E$ over $K$ and an integer $n>1$. Suppose that $n \cdot y=f$ with $f \in E(K)$ has a solution $y_{v}$ in every $E\left(K_{v}\right)$. Does there exists a solution $y \in E(K)$ ? By "folklore" the answer is positive and the analogous problem for Drinfeld modules, where the integer $n$ is replaced by a nonzero element of $\mathbb{F}_{q}[z]$, has a negative answer (see [Van der Heiden 2005] for both statements).

Here we consider a differential operator $L=a_{n} \partial^{n}+\cdots+a_{1} \partial+a_{0}$, where $\partial$ is the derivation on $K$ extending $d / d z$ on $\mathbb{C}(z)$ and $a_{n}, \ldots, a_{0} \in K$, acting upon $K$. Suppose that the equation $L(y)=f$ with $f \in K$ has a solution $y_{v}$ in every completion $K_{v}$. Then, in general, there is no solution in $K$. One defines the $\mathbb{C}$-vector space $\lg l(L)$ as the kernel of the obvious $\mathbb{C}$-linear map $K / L(K) \rightarrow \prod_{v} K_{v} / L\left(K_{v}\right)$. This vector space measures this local-global problem.

MSC2000: primary 34A30; secondary 34M15.

Keywords: Galois differential groups, abelian differential extensions, local and global solutions of differential equations. 
The theme of this paper is the interpretation and the computation of $\operatorname{lgl}(L)$. The main results are

- a formula expressing $\lg l(L)$ in terms of the cohomology for differential Galois groups acting on the solution space of $L$, and a proof of $\operatorname{dim} \operatorname{lgl}(L)<\infty$;

- computation of $H^{*}(G, V)$ for certain affine group schemes $G$;

- classification of abelian differential equations and Artin reciprocity;

- explicit computations of $\lg l(L)$ for abelian differential operators and for regular singular operators $L$;

- a new proof of B. Malgrange's results on irregularity.

The last item requires a precise knowledge of the universal differential Galois group for the differential field $F=\mathbb{C}(\{z\})$ of the convergent Laurent series. The multisummation theory of J.-P. Ramis et al. provides this knowledge.

This kind of problem, local global for differential equations, has been previously studied by J. Manin [1965].

\section{2. $K / L(K)$ and $M / \partial M$ as cohomology groups}

Let $K$ denote a differential field, and let $\partial(f)$ or $f^{\prime}$ denote the derivative of $f \in K$. We suppose that its field of constants $C:=\left\{f \mid f^{\prime}=0\right\}$ is algebraically closed and different from $K$ and has characteristic 0 . A linear differential equation over $K$ can be written in an operator form

$$
\left(a_{n} \partial^{n}+\cdots+a_{1} \partial+a_{0}\right) y=f \quad \text { with } a_{i}, f \in K .
$$

An equivalent formulation is given by a differential module $M=(M, \partial)$, where $M$ is a finite dimensional vector space over $K$ and the additive operator $\partial: M \rightarrow M$ satisfies $\partial(f m)=f^{\prime} m+f \partial m$. (The meaning of the symbols $\partial$ and ' will be clear from the context.)

We recall (see for details [Van der Put and Singer 2003, Chapters 1 and 2]) that for every linear differential equation $M$ (or module) over $K$, there is a differential ring $\operatorname{PVR}(M / K)$, called the Picard-Vessiot ring of $M$ over $K$, such that all solutions of $\partial m=0$ live in this differential ring and this ring has only trivial differential ideals. The (differential) Galois group of the module $M$ is the linear algebraic group over $C$ consisting of all $K$-linear automorphisms of $\operatorname{PVR}(M / K)$ commuting with the differentiation on $\operatorname{PVR}(M / K)$. The direct limit of all $\operatorname{PVR}(M / K)$ is the universal differential extension $U(K)$ of $K$. Its Galois group $G(K, \partial)$ is the affine group scheme, which is the projective limit of the Galois groups of all differential modules $M$ over $K$.

For a differential operator $L \in K[\partial], L \neq 0$, the solution space of $L$ is the $C$-vector space $V(L):=\operatorname{ker}(L, U(K))$ is the solution space of $L$. The action 
of $G(K, \partial)$ on $U(K)$ leaves $V(L)$ invariant, and the restriction of the action to $V$ is the (differential) Galois group of $L$. Similarly, for a differential module $M$ over $K$, the $C$-vector space $V(M):=\operatorname{ker}\left(\partial, U(K) \otimes_{K} M\right)$ is the solution space of $M$, and the restriction to $V(M)$ of the natural action of $G(K, \partial)$ on $U(K) \otimes_{K} M$ is the (differential) Galois group of $M$.

Proposition 2.1. We have $H^{0}(G(K, \partial), U(K))=K$ and $H^{i}(G(K, \partial), U(K))=0$ for all $i \geq 1$.

Proof. Using that $U(K)$ is a direct limit of Picard-Vessiot rings, one concludes that it suffices to consider a differential module $M$ over $K$ with Picard-Vessiot ring $L \supset K$ and Galois group $G$. In this case one has to prove $H^{0}(G, L)=L^{G}=K$ and $H^{i}(G, L)=0$ for all $i \geq 1$.

The first statement is well known. The affine variety corresponding to $L$ is known to be a $K$-torsor for the linear algebraic group $G$ over $C$. In other words, there exists a finite Galois extension $K^{+} \supset K$ such that $K^{+} \otimes_{K} L \cong K^{+} \otimes_{C} C[G]$. Further the action of the Galois group $\operatorname{Gal}\left(K^{+} / K\right)$ on this object commutes with the action of $G$.

We recall that the cohomology groups $H^{i}(G, V)$, where $V$ is any $G$-module, are the cohomology groups of the Hochschild complex $C^{*}(G, V)$. Moreover, one has $H^{i}(G, C[G])=0$ for all $i \geq 1$, since $C[G]=\operatorname{Ind}_{\{1\}}^{G} C$ is an injective module (see [Jantzen 2003] for these statements). It follows that also $H^{i}\left(G, K^{+} \otimes_{C} C[G]\right)=0$ for $i \geq 1$. For such $i$, let $\xi$ be an element in $C^{i}(G, L)$ with $d^{i} \xi=0$. Then $\xi=d^{i-1} \eta$ for some $\eta \in C^{i-1}\left(G, K^{+} \otimes L\right)$. Then

$$
\eta^{*}:=\frac{1}{\# \operatorname{Gal}\left(K^{+} / K\right)} \cdot \sum_{\sigma \in \operatorname{Gal}\left(K^{+} / K\right)} \sigma(\eta)
$$

belongs to $C^{i-1}(G, L)$ and satisfies $d^{i-1} \eta^{*}=\xi$.

Lemma 2.2. Let $L \in K[\partial]$ be a nonzero differential operator and $U(K), G(K, \partial)$ be as before. The following sequences are exact:

$$
\begin{aligned}
& 0 \rightarrow \operatorname{ker}(L, U(K)) \longrightarrow U(K) \stackrel{L}{\longrightarrow} U(K) \rightarrow 0 \text { and } \\
& 0 \rightarrow \operatorname{ker}(L, K) \longrightarrow K \stackrel{L}{\longrightarrow} K \longrightarrow H^{1}(G(K, \partial), \operatorname{ker}(L, U)) \rightarrow 0 .
\end{aligned}
$$

In particular, $K / L(K)$ is canonically isomorphic to $H^{1}(G(K, \partial), V(L))$, and also $H^{i}(G(K, \partial), V(L))=0$ for $i \geq 2$.

This lemma could be proved by the arguments of [Bertrand 1990, first paragraph of p. 126, Remark 1 p. 217, and p. 131]. However, the lemma does not appear explicitly there. 
Proof. For the exactness of the first sequence we must show that $L y=f$ with $f \in U(K)$ has a solution $y \in U(K)$. For any $f \in U(K)$ there exists a nonzero $\tilde{L} \in K[\partial]$ such that $\tilde{L} f=0$. Indeed, since $f$ lies in some $\operatorname{PVR}(M)$, apply now [Van der Put and Singer 2003, Corollary 1.38]. The equation $\tilde{L}(L(y))=0$ has all its solutions in $U(K)$. Hence for a suitable solution $y \in U(K)$ of this equation, one has $L y=f$.

Taking, in the first exact sequence, invariants under $G(K, \partial)$, one obtains, by using Proposition 2.1, the exact sequence

$$
0 \rightarrow \operatorname{ker}(L, K) \longrightarrow K \stackrel{L}{\longrightarrow} K \longrightarrow H^{1}(G, \operatorname{ker}(L, U(K))) \rightarrow 0 .
$$

For a differential module $M$ over $K$ one has a similar result that there is a canonical isomorphism $M / \partial M \rightarrow H^{1}(G(K, \partial), V(M))$, where again $V(M)=$ $\operatorname{ker}\left(\partial, U(K) \otimes_{K} M\right)$, provided with its $G(K, \partial)$-module structure.

A direct comparison between modules and differential operators is given by the theorem of the cyclic element, which states that any differential module $M$ has the form $K[\partial] / K[\partial] L$ for some operator $L$. Let $L^{*}$ denote the adjoint of $L$. Then one can verify that $V(M)$ can be identified with $V\left(L^{*}\right)$ and $M / \partial M$ with $K / L^{*}(K)$.

The use of cohomology of groups to study differential operators or modules is already present in D. Bertrand's papers [1990; 1992]; see also [Hardouin 2005].

\section{On cohomology of linear algebraic groups}

The base field $k$ is assumed to be algebraically closed and to have characteristic 0 . Let $G$ be a linear algebraic group, or more generally an affine group scheme, over $k$. A $G$-module $V$ is a finite-dimensional vector space over $k$ provided with an algebraic action of $G$, that is, a morphism of affine group schemes $\rho: G \rightarrow \operatorname{GL}(V)$. The cohomology groups $H^{*}(G, V)$ are defined as the derived functors of $V \mapsto V^{G}$. The $k$-vector space $H^{i}(G, V)$ can, as in the case of ordinary group cohomology, be described as the space of all $i$-cocycles $f: G \times \cdots \times G \rightarrow V$, divided out by the subspace of the trivial $i$-cocycles. The only difference is that the $i$-cocycles $f$ are supposed to be morphisms of algebraic varieties (or more generally, of affine schemes). We will also allow $G$-modules $V$ of infinite dimension, namely direct limits of finite-dimensional $G$-modules. Now we collect here (with some comments) the facts and results that we will need in the sequel and refer to [Jantzen 2003] for the general theory.

Fact 3.1. Let $G$ be a reductive affine group scheme (not necessarily connected). Then for every $G$-module $V$, one has $H^{i}(G, V)=0$ for all $i \geq 1$.

Indeed, since the characteristic of $k$ is 0 , the functor $V \mapsto V^{G}$ is exact. 
Fact 3.2. Let $N$ be a closed, normal subgroup of the affine group scheme $G$. Suppose that $G / N$ is reductive. Let $V$ be a $G$-module. Then $H^{*}(G, V)$ is canonically isomorphic to $H^{*}(N, V)^{G / N}$.

Indeed, the functor $V \mapsto V^{G}$ factors as $H^{0}\left(G / N, V^{N}\right)$; the functor $H^{0}(G / N, \cdot)$ is exact and maps injective objects to acyclic objects. The special case of Fact 3.2 in which $N$ is the unipotent radical $R_{u}(G)$ reduces the computations to the case of (connected) unipotent groups.

Fact 3.3 (the five term exact sequence). Let $N$ be a closed normal subgroup of the affine group $G$, and let $V$ be a $G$-module. Then the exact sequence of five terms reads

$$
\begin{aligned}
0 \rightarrow H^{1}\left(G / N, V^{N}\right) \longrightarrow & H^{1}(G, V) \\
& \longrightarrow H^{1}(N, V)^{G / N} \longrightarrow H^{2}\left(G / N, V^{N}\right) \longrightarrow H^{2}(G, V) .
\end{aligned}
$$

This exact sequence is derived from the spectral sequence $H^{a}\left(G / N, H^{b}(N, V)\right)$ converging to $H^{a+b}(G, V)$.

Remark 3.4 (explicit action of $G / N$ on $H^{1}(N, V)$ ). Let $V$ be a $G$-module and $N$ a normal closed subgroup of $G$. The action of $G / N$ on $H^{1}(N, V)$ can be made explicit on the level of 1-cocycles. Let $f: N \rightarrow V$ be a 1-cocycle and $g \in G$. Then $(g f)$ is the 1-cocycle defined by $(g f)(n)=g f\left(g^{-1} n g\right)$. The trivial 1-cocycle $f(n)=n v-v$ for a fixed $v \in V$ is mapped to the trivial 1-cocycle $n \mapsto n(g v)-(g v)$. Thus $G$ acts on $H^{1}(N, V)$. For $m \in N$ and a 1-cocycle $f: N \rightarrow V$, one observes that $(m f)-f$ is the trivial 1-cocycle $n \mapsto n f(m)-f(m)$. Thus $N$ acts trivially on $H^{1}(N, V)$.

Fact 3.5. Let $\mathfrak{g}$ be the Lie algebra of a connected affine group scheme $G$. Any $G$-module $V$ has the structure of a $\mathfrak{g}$-module. The cohomology groups $H^{*}(G, V)$ are canonically isomorphic to the cohomology groups $H^{*}(\mathfrak{g}, V)$.

Sketch of the proof. Using Fact 3.2, we may suppose that $G$ is a connected unipotent linear algebraic group over $\mathbb{C}$. In particular, $G$ is simply connected. Therefore the category of the finite-dimensional representations of $G$ and that of its Lie algebra $\mathfrak{g}$ are equivalent. This equivalence extends to an equivalence between the representations of $G$ that are direct limits of finite-dimensional representations and those of $\mathfrak{g}$. The latter categories contain enough injective objects, and the cohomology groups can be obtained from injective resolutions.

We note that the group $H^{i}(\mathfrak{g}, V)$ can also be described by $i$-cocycles modulo trivial $i$-cocycles; see [Jacobson 1962] and [Varadarajan 1984, Section 3.12]. In some cases the computations of the $H^{*}(\mathfrak{g}, V)$ are easier than those for $H^{*}(G, V)$. 
Lemma 3.6. Let $t$ be a generator of the Lie algebra of $\mathbb{G}_{a}$. The $\mathbb{G}_{a}$-module $V$, given by $\rho: \mathbb{G}_{a} \rightarrow \mathrm{GL}(V)$, induces a nilpotent map $\rho(t) \in \operatorname{End}(V)$. Then

$$
\begin{aligned}
& H^{0}\left(\mathbb{G}_{a}, V\right)=\operatorname{ker}(\rho(t), V)=\operatorname{ker}(\exp (\rho(t))-1, V), \\
& H^{1}\left(\mathbb{G}_{a}, V\right)=\operatorname{coker}(\rho(t), V)=\operatorname{coker}(\exp (\rho(t))-1, V), \\
& H^{i}\left(\mathbb{G}_{a}, V\right)=0 \quad \text { for } i \geq 2 .
\end{aligned}
$$

Proof. By Fact 3.5, it suffices to compute the cohomology of $V$ as a module over the Lie algebra of $\mathbb{G}_{a}$. An obvious calculation of these cohomology groups in terms of cocycles gives the required answer. The same computation can be done in terms of cocycles for the group $\mathbb{G}_{a}$.

Corollary 3.7. Let the group scheme $G$ be topologically generated by one element $A$. Suppose the unipotent factor $A_{u}$ of the Jordan decomposition $A=A_{s s} \cdot A_{u}$ is nontrivial. Then $G=G_{1} \times \mathbb{G}_{a}$, where the first factor is topologically generated by $A_{s s}$ and the second by $A_{u}$.

Let the $G$-module $V$ be given by $\rho: G \rightarrow \operatorname{GL}(V)$. Then $H^{i}(G, V)=0$ for $i \geq 2$, and $H^{1}(G, V)=\operatorname{coker}\left(\rho\left(A_{u}\right)-1, V^{G_{1}}\right)=\operatorname{coker}(\rho(A)-1, V)$.

Proof. $H^{*}(G, V)=H^{*}\left(\mathbb{G}_{a}, V\right)^{G_{1}}$, and this equals $H^{*}\left(\mathbb{G}_{a}, V^{G_{1}}\right)$ since $G_{1}$ commutes with $\mathbb{G}_{a}$. Apply now Lemma 3.6. The final statement follows from the decomposition of $V$ into eigenspaces for $\rho\left(A_{s s}\right)$.

3.8. Group schemes with free unipotent generators. Let $S$ be some nonempty set. One considers tuples $(V, \alpha)$, where $V$ is a finite-dimensional $k$-vector space and $\alpha: S \rightarrow \mathrm{GL}(V)$ maps every $s \in S$ to a unipotent $\alpha(s)$. This defines an abelian category Unipotent $(S)$ which is in an obvious way a Tannakian category. The fiber functor is given by $(V, \alpha) \mapsto V$. Thus $\operatorname{Unipotent}(S)$ is isomorphic to the category of the finite-dimensional representations of a certain affine group scheme $N$ over $k$.

Consider an object $(V, \alpha)$ and the Tannakian subcategory $\{\{(V, \alpha)\}\}$ generated by it. The affine group scheme corresponding to this subcategory can be seen to be the smallest algebraic subgroup $H(V, \alpha)$ of GL $(V)$ containing all $\alpha(s)$. The representation $\rho: N \rightarrow \mathrm{GL}(V)$, corresponding to $(V, \alpha)$, has the property that $\rho(N)=H(V, \alpha)$. In particular, if $\rho(N)$ is a finite group, then $\rho(N)=\{1\}$. Thus $N$ is a connected affine group scheme. Moreover, $N$ is the projective limit of the $H(V, \alpha)$, taken over all objects $(V, \alpha)$. For a fixed $s \in S$, each $H(V, \alpha)$ contains an element $\alpha(s)$. The projective limit of the elements $\alpha(s) \in H(V, \alpha)$ can be considered as an element, again called $s$, in $N$. Thus $\rho(s)=\alpha(s)$ for every object $(V, \alpha)$ and corresponding representation $\rho: N \rightarrow \operatorname{GL}(V)$. Therefore, we will call $N$ the group with free unipotent generators $S$. Indeed, the elements of $S$, seen as elements of $N$, are topological generators; they are unipotent and have no relations. 
We want to show that for any $N$-module $V$, the $H^{*}(N, V)$ are the cohomology groups of the complex $0 \rightarrow V \rightarrow V^{S} \rightarrow 0$, where the nontrivial map is given by $v \mapsto(\rho(s) v-v)_{s \in S}$. Though a direct proof is perhaps, we will prove this using the (pro-)Lie algebra of $N$.

3.9. Lie algebras with free nilpotent generators. Let $S$ be again a nonempty set. The free Lie algebra $F$ over $k$ with generators $S \subset F$ has the universal property that the representations $\tau: F \rightarrow \operatorname{End}(V)$ on vector spaces $V$ over $k$ are in bijection with the maps $S \rightarrow \operatorname{End}(V)$. One considers now a representation $\tau$ such that $V$ is finite-dimensional and every $\tau(s)$ is nilpotent. The image $F(\tau) \subset \operatorname{End}(V)$ is an algebraic Lie algebra, because it is generated by nilpotent maps. The corresponding (connected) algebraic subgroup of GL( $V)$ is the smallest algebraic subgroup containing all $\exp (\tau(s))$. Define $\rho: S \rightarrow \operatorname{GL}(V)$ by $\rho(s)=\exp (\tau(s))$. Then $(V, \rho)$ is an object of Unipotent $(S)$, the Tannakian group of this object is the smallest algebraic group containing all $\exp (\tau(s))$, and its Lie algebra is $F(\tau)$.

The projective $\operatorname{limit}_{\leftarrow} \lim _{\leftarrow} F / \operatorname{ker} \rho$ taken over all these $\rho$ will be called the (pro-)Lie algebra with free nilpotent generators $S$. It is clear from the above that this pro-Lie algebra is the Lie algebra of the affine group scheme $N$ of Section 3.8. The bijection between the representations of $N$ and those of its (pro)-Lie algebra can be interpreted as $N$ being simply connected.

Proposition 3.10. Let $N$ be the affine group scheme with free unipotent generators $S$, and let $V$ be a $N$-module. The $H^{*}(N, V)$ are the cohomology groups of the complex $0 \rightarrow V \rightarrow V^{S} \rightarrow 0$, where the nontrivial map $V \rightarrow V^{S}$ is given by $v \mapsto(\rho(s) v-v)_{s \in S}$.

Proof. Let Lie $(N)$ denote the pro-Lie algebra of $N$. According to Fact 3.5 and the constructions in Sections 3.8 and 3.9, the proposition is equivalent to the statement that the $H^{*}(\operatorname{Lie}(N), V)$ are the cohomology groups of the complex $0 \rightarrow V \rightarrow$ $V^{S} \rightarrow 0$, where the nontrivial map is given by $v \mapsto(\log (\rho(s)) v)_{s \in S}$.

For $H^{0}(\operatorname{Lie}(N), V)$, this is obvious. For $H^{1}(\operatorname{Lie}(N), V)$ we use the explicit definition of a 1-cocycle $f$ (see [Jacobson 1962] and [Varadarajan 1984, Section 3.12]) and conclude that the elements $f(s) \in V$ are arbitrary and that they determine $f$ completely. The trivial 1-cocycles are of the form $f(z)=\tau(z) v$ for a fixed $v \in V$, where $\tau: \operatorname{Lie}(N) \rightarrow \operatorname{End}(V)$ is induced by $\rho: N \rightarrow \operatorname{GL}(V)$. This proves the claim for $H^{1}(\operatorname{Lie}(N), V)$. The verification of $H^{i}(\operatorname{Lie}(N), V)=0$ for $i \geq 2$ is easy.

3.11. Finiteness conditions. Let the set $S$ be a disjoint union of (nonempty) subsets $S_{i}$ with $i \in I$ (and $I$ an infinite set). Let Unipotent $\left(\left\{S_{i}\right\}_{i \in I}\right)$ denote the category for which the objects are the pairs $(V, \alpha)$ with $V$ a finite-dimensional $k$-vector space and $\alpha: S \rightarrow \mathrm{GL}(V)$ such that $\alpha(s)$ is unipotent for every $s \in S$ and there is a finite subset $J$ of $I$ such that $\alpha(s)=1$ for $s \notin \bigcup_{i \in J} S_{i}$. As in Sections 3.8 and 3.9, this 
defines an affine group scheme $N$ and a pro-Lie algebra $\operatorname{Lie}(N)$. The analogue of Proposition 3.10 is as follows:

Proposition. Let $V$ be an $N$-module. The $H^{*}(N, V)$ are the cohomology groups of the complex $0 \rightarrow V \rightarrow V^{(S)} \rightarrow 0$, where $V^{(S)}$ denotes the $k$-vector space of the maps $f: S \rightarrow V$ such that there exists a finite subset $J$ of $I$ with $f(s)=0$ for $s \notin \bigcup_{i \in J} S_{i}$. The nontrivial map is again $v \mapsto(\rho(s) v-v)_{s \in S}$.

The above situation occurs in connection with the Stokes phenomenon [Van der Put and Singer 2003, Chapter 8], where $S=\left\{\Delta_{q, d} \mid q \in 2, d\right.$ is singular for $\left.q\right\}$ is the set of alien derivations. The set $S$ is the disjoint union, over $q \in 2$, of the sets $S_{q}:=\{d \in \mathbb{R} \mid d$ is singular for $q\}$. The corresponding affine group scheme $N$ is the kernel of the surjective morphism of affine schemes $G_{\text {analytic }} \rightarrow G_{\text {formal }}$. We will return to this in Section 5. We note that the finiteness condition stated in [Van der Put and Singer 2003, Chapter 10, p. 268] is slightly wrong.

\section{Formal differential equations}

Let $\widehat{F}$ be the differential field $\mathbb{C}((z))$ with derivation $\delta:=z \frac{d}{d z}$. A differential equation or module over $\widehat{F}$ will be called formal. We recall the explicit descriptions of $U(\widehat{F})$ and $G_{\text {formal }}:=G(\widehat{F}, \partial)$, slightly extending the one given in [Van der Put and Singer 2003, Chapter 3, Section 3.2]. The aim of this section is to make explicit both $H^{*}\left(G_{\text {formal }}, V(M)\right)$ (where $V(M)$ is the solution space of $M$ ) and the canonical isomorphism $M / \partial M \rightarrow H^{1}\left(G_{\text {formal }}, V(M)\right)$.

Description of $\boldsymbol{U}(\widehat{\boldsymbol{F}})$. Write $\mathbb{C}=A \oplus \mathbb{Q}$ with $A$ a $\mathbb{Q}$-vector space. First one introduces the universal Picard-Vessiot ring $U_{\mathrm{rs}}$ for the regular singular differential modules over $\widehat{F}$. This ring is $U_{\mathrm{rs}}:=\widehat{F}_{\mathrm{cl}}\left[\{e(c)\}_{c \in A}, \ell\right]$, where $\widehat{F}_{\mathrm{cl}}$ is the algebraic closure of $\widehat{F}$ and where the symbols $e(c)$ and $\ell$ satisfy only the identities $e\left(c_{1}+c_{2}\right)=e\left(c_{1}\right) \cdot e\left(c_{2}\right)$. The differentiation is given by $\delta e(c)=c \cdot e(c)$ and $\delta \ell=1$. Then one introduces the set $2=\bigcup_{m \geq 1} z^{-1 / m} \mathbb{C}\left[z^{-1 / m}\right]$ and symbols $e(q)$ for $q \in 2$ satisfying only the identities $e\left(q_{1}+q_{2}\right)=e\left(q_{1}\right) \cdot e\left(q_{2}\right)$. Further $\delta e(q)=q \cdot e(q)$. Now $U(\widehat{F}):=\bigoplus_{q \in 2} U(\widehat{F}, q)$, where $U(\widehat{F}, q):=U_{\text {rs }} e(q)$. This is the universal Picard-Vessiot ring for $\widehat{F}$. Put $2^{+}:=2 \oplus A$ and write $e(q+c):=e(q) \cdot e(c)$ for $q+c \in 2^{+}$. Then $U(\widehat{F})=\widehat{F}_{\mathrm{cl}}\left[\{e(q)\}_{q \in \mathcal{2}^{+}}, \ell\right]$.

Description of $\boldsymbol{G}_{\text {formal. }}$. For convenience, we will identify the affine group scheme $G_{\text {formal }}$ with its set of $\mathbb{C}$-valued points, which is the group of the differential automorphisms of $U(\widehat{F}) / \widehat{F}$. A special (and very natural) element in this group is the formal monodromy $\gamma$ defined by the properties

(i) $\gamma$ acts on $\widehat{F}_{\mathrm{cl}}$ by $\gamma\left(z^{\lambda}\right)=e^{2 \pi i \lambda} z^{\lambda}$ for all $\lambda \in \mathbb{Q}$,

(ii) $\gamma e(c)=e^{2 \pi i c} e(c)$ for all $c \in A$, 
(iii) $\gamma(e(q))=e(\gamma q)$ for all $q \in 2$, and

(iv) $\gamma(\ell)=\ell+2 \pi i$.

In J.-P. Ramis's terminology, the exponential torus $T$ is the group $\operatorname{Hom}\left(2, \mathbb{C}^{*}\right)$. An element $h \in T$ that acts on $U(\widehat{F})$ by $h$ is the identity on $\widehat{F}_{\mathrm{cl}}$, on $\ell$, and on the elements $e(c)$. Further $h(e(q))=h(q) \cdot e(q)$ for all $q \in 2$. The group $T$ together with the element $\gamma$ topologically generate $G_{\text {formal }}$ (for the Zariski topology). So far we have followed [Van der Put and Singer 2003, Chapter 10]. The Zariski closure $\overline{\langle\gamma\rangle}$ of the group $\langle\gamma\rangle$ generated by $\gamma$ is rather big, and we prefer to split this group into smaller pieces. For this purpose we introduce more special elements in $G_{\text {formal }}$.

One decomposes $\gamma$ as a product of commuting automorphisms $\gamma_{s s} \gamma_{u}$, where $\gamma_{s s}$ has the same definition as $\gamma$ except for (iv), which is replaced by $\gamma_{s s} \ell=\ell$. Further $\gamma_{u}$ is the identity for the elements in $\widehat{F}_{\mathrm{cl}}$, the elements $e(c), e(q)$ and $\gamma_{u} \ell=$ $\ell+2 \pi i$. We note that $\gamma_{s s} \gamma_{u}$ is the Jordan decomposition of $\gamma$ as a product of a semisimple element and a unipotent element.

We still want to decompose the semisimple $\gamma_{s s}$ as a product of commuting elements $\gamma_{0}$ and $\gamma_{1}$. The direct sum $\mathbb{C}=\mathbb{Q} \oplus A$ yields, using $c \mapsto e^{2 \pi i c}$, a direct product decomposition $\mathbb{C}^{*}=\left(\mu_{\infty}\right) \times e^{2 \pi i A}$. Now $\gamma_{0}$ and $\gamma_{1}$ are the unique semisimple elements in $G_{\text {formal }}$ with eigenvalues in $\mu_{\infty}$ and $e^{2 \pi i A}$ such that $\gamma_{0} \gamma_{1}=\gamma_{s s}$. One verifies that $\gamma_{0}$ and $\gamma_{1}$ can also be defined by

(i) $\gamma_{0}\left(z^{\lambda}\right)=e^{2 \pi i \lambda} z^{\lambda}$ and $\gamma_{1}\left(z^{\lambda}\right)=z^{\lambda}$ for all $\lambda \in \mathbb{Q}$,

(ii) $\gamma_{0}(e(q))=e\left(\gamma_{0} q\right)$ and $\gamma_{1} e(q)=e(q)$ for all $q \in 2$,

(iii) $\gamma_{0}(e(c))=e(c)$ and $\gamma_{1} e(c)=e^{2 \pi i c} e(c)$ for all $c \in A$, and

(iv) $\gamma_{0}(\ell)=\gamma_{1}(\ell)=\ell$.

For every element $a \in U(\widehat{F})$, there is an integer $n \geq 1$ such that $\gamma_{0}^{n}(a)=a$. It follows that the algebraic subgroup of $G_{\text {formal }}$ generated (topologically) by $\gamma_{0}$ is $\widehat{\mathbb{Z}}$, the projective limit of the groups $\mathbb{Z} / n \mathbb{Z}$. The algebraic subgroup generated (topologically) by $\gamma_{1}$ can be identified with the torus $\operatorname{Hom}\left(A, \mathbb{C}^{*}\right)$. The algebraic subgroup generated by $\gamma_{u}$ can be identified with $\mathbb{G}_{a}$.

Thus $\overline{\langle\gamma\rangle}$, the Zariski closure of the group generated by $\gamma$, can be identified with $\mathbb{G}_{a} \times \operatorname{Hom}\left(A, \mathbb{C}^{*}\right) \times \widehat{\mathbb{Z}}$. Moreover, $\gamma$ is the topological generator of $G_{\mathrm{rs}}$, the group of the differential automorphisms of $U_{\mathrm{rs}} / \widehat{F}$ or, in other words, the universal differential Galois group for the regular singular equations over $\widehat{F}$.

We extend the exponential torus $T$ to a larger torus $T^{+}=\operatorname{Hom}\left(2^{+}, \mathbb{C}^{*}\right)$. An element $h \in T^{+}$that acts on $U(\widehat{F})$ by $h$ is the identity on $\widehat{F}_{\mathrm{cl}}$ and $\ell$. Further $h(e(q)):=h(q) \cdot e(q)$ for $q \in 2^{+}$. 
We conclude that $G_{\text {formal }}$ has the form $\mathbb{G}_{a} \times\left(T^{+} \rtimes \widehat{\mathbb{Z}}\right)$. The latter subgroup $T^{+} \rtimes \widehat{\mathbb{Z}}$ is reductive, and the subgroup $\mathbb{G}_{a}$ is the unipotent radical of $G_{\text {formal }}$. Also $G_{\text {formal }} / G_{\text {formal }}^{\text {o }}=\widehat{\mathbb{Z}}$ with topological generator $\gamma_{0}$.

Description of differential modules over $\widehat{\boldsymbol{F}}$. One associates to a differential module $M$ over $\widehat{F}$ its solution space, defined as $V=V(M):=\operatorname{ker}\left(\partial, U(\widehat{F}) \otimes_{\widehat{F}} M\right)$ with the data of (i) a decomposition $V=\bigoplus_{q \in 2} V_{q}$, where $V_{q}=\operatorname{ker}\left(\partial, U(\widehat{F}, q) \otimes_{F} M\right)$, (ii) an element $\gamma_{V} \in \mathrm{GL}(V)$, the restriction of $\gamma$ on $U(\widehat{F}) \otimes_{\widehat{F}} M$ to the subspace $V$, and relations (iii) $\gamma_{V}\left(V_{q}\right)=V_{\gamma q}$ for all $q \in 2$.

The functor $M \mapsto V(M):=\left(V,\left\{V_{q}\right\}, \gamma_{V}\right)$ defines an equivalence of categories. We note that $\gamma_{V}$ has a decomposition as a product $\gamma_{V, 0} \gamma_{V, 1} \gamma_{V, u}$ of commuting elements induced by $\gamma=\gamma_{0} \gamma_{1} \gamma_{u}$.

Computation of $\boldsymbol{H}^{\mathbf{1}}\left(\boldsymbol{G}_{\text {formal }}, \boldsymbol{V}(\boldsymbol{M})\right)$. By Section $3, H^{i}\left(G_{\text {formal }}, V\right)=H^{i}\left(\mathbb{G}_{a}, W\right)$ with $W:=V^{T^{+} \rtimes \widehat{\mathbb{Z}}}$. From the above one sees that $W=V_{0}^{\gamma_{s s}}$. Now $\widehat{F}[\ell]$ is the subspace of $U(\widehat{F})$ consisting of the elements invariant under $T^{+} \rtimes \widehat{\mathbb{Z}}$. Hence $W=$ $\operatorname{ker}\left(\partial, \widehat{F}[\ell] \otimes_{\widehat{F}} M\right)$. The action of $\gamma_{u}$ on $W$ is induced by its action on $\widehat{F}[\ell]$, given by $\ell \mapsto \ell+2 \pi i$. The group $\mathbb{G}_{a}$, generated for the Zariski topology by $\gamma_{u}$, acts on $W$ by $t \mapsto \gamma_{u, V}^{t}$. Hence $H^{i}\left(G_{\text {formal }}, V(M)\right)$ can be identified with $\operatorname{ker}\left(\log \gamma_{u, V}, W\right)$ for $i=0$ and with coker $\left(\log \gamma_{u, V}, W\right)$ for $i=1$. In particular, the two cohomology groups have the same dimension. This proves the following corollary.

Corollary 4.1 [Malgrange 1974]. For any differential module $M$ over $\widehat{F}$, one has $\operatorname{dim}_{\mathbb{C}} \operatorname{ker}(\partial, M)=\operatorname{dim}_{\mathbb{C}} M / \partial M$.

The explicit canonical isomorphism can : $M / \partial M \rightarrow H^{\mathbf{1}}\left(G_{\text {formal }}, V(M)\right)$. Any module $M$ is, after a finite extension of $\widehat{F}$, a direct sum of isotypical submodules modules $M(q)$ with $q \in 2$. This is just a translation of the decomposition $V=$ $\bigoplus V_{q}$. Now $\partial$ is bijective on $M(q)$ for $q \neq 0$. Thus for $q \neq 0$, the summand $M(q)$ gives no contribution for the above map can. The direct summand $M(0)$, which is the regular singular part of $M$, can be represented by a matrix differential operator $z \frac{d}{d z}+B$ with $B$ a constant matrix whose eigenvalues $\lambda$ satisfy $0 \leq \operatorname{Re}(\lambda)<1$. Only the generalized eigenspace for the eigenvalue 0 of $B$ can give a contribution to $M(0) / \partial M(0)$. This generalized eigenspace is the submodule of $M(0) \approx \widehat{F}^{d}$ corresponding to $W=V_{0}^{\gamma_{s s}}$. After restricting to this submodule, $B$ is nilpotent. The kernel and cokernel of $z \frac{d}{d z}+B$ on $\widehat{F}^{d}$ coincide with the kernel and cokernel of $B$ on $\mathbb{C}^{d}$. The fundamental matrix for the equation is $e^{-B \log z}$. The columns of this matrix form a basis for $\operatorname{ker}\left(\partial, \widehat{F}[\ell] \otimes_{\widehat{F}} M\right)$. The differential Galois group is generated by the action of $\gamma_{u}$ that multiplies the fundamental matrix by $e^{-2 \pi i B}$. Thus $\log \gamma_{u}$ on $W$ can be identified with $-B /(2 \pi i)$ on $\mathbb{C}^{d}$. 


\section{Analytic differential equations}

$F$ denotes the differential field $\mathbb{C}(\{z\})$ consisting of the convergent Laurent series, with derivation $\delta:=z \frac{d}{d z}$. A differential equation or module over this field will be called analytic. We recall and extend results of [Van der Put and Singer 2003, Chapters 8, 9 and 10].

Description of $\boldsymbol{U}(\boldsymbol{F})$ and $\boldsymbol{G}_{\text {analytic }}:=\boldsymbol{G}(\boldsymbol{F}, \partial)$. As in Section $4, \widehat{F} \supset F$ denotes the field of the formal Laurent series. Let $\mathscr{D} \subset \widehat{F}_{\mathrm{cl}}$ denote the $\bar{F}$-subalgebra consisting of the elements $h$ that satisfy a linear differential equation with coefficients in $\bar{F}$. Then

$$
\bar{F}\left[\{e(q)\}_{q \in \mathscr{2}^{+}}, \ell\right] \subset U(F)=\mathscr{D}\left[\{e(q)\}_{q \in \mathscr{2}^{+}}, \ell\right] \subset U(\widehat{F})=\widehat{F}_{\mathrm{cl}}\left[\{e(q)\}_{q \in \mathscr{2}^{+}} . \ell\right] .
$$

The precise structure of the differential algebra $\mathscr{D}$ is unknown. However the multisummations $\left\{\text { mult }_{d}\right\}_{d \in \mathbb{R}}$ in the directions $d$ lead to "locally unipotent" elements of $G_{\text {analytic }}$, the Stokes maps (multipliers) for every direction $d$. One considers the "locally nilpotent" logarithms $\Delta_{d}$ of the Stokes maps. These are elements

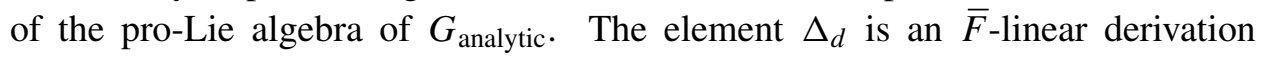
of $U(F)$, commuting with $\delta$, and trivial on $\bar{F}\left[\{e(q)\}_{q \in 2^{+}}, \ell\right]$. Thus $\Delta_{d}$ is determined by its restriction $\Delta_{d}: \mathscr{D} \rightarrow U(F)=\bigoplus_{q \in 2} U(F, q)$. The resulting maps $\Delta_{q, d}: \mathscr{D} \rightarrow U(F, q)$ are important ingredients for the structure of $G_{\text {analytic. }}$ Put $\left\{x_{i}\right\}_{i \in I}=\left\{\Delta_{q, d} \mid d\right.$ singular for $\left.q\right\}$, and let $\operatorname{Lie}(N)$ and $N$ denote the proLie algebra and the affine group scheme corresponding to $\left\{x_{i}\right\}$ defined in Section 3. Then $G_{\text {analytic }}=N \rtimes G_{\text {formal }}$. We recall that $G_{\text {formal }}$ is topologically generated by $T=\operatorname{Hom}\left(2, \mathbb{C}^{*}\right)$ and the formal monodromy $\gamma$. The action, by conjugation, of $G_{\text {formal }}$ on $N$ induces an action on $\operatorname{Lie}(N)$. One can verify that $\gamma\left(\Delta_{q, d}\right)=\Delta_{\gamma(q), d-2 \pi}$ and that, for any $h \in T$, one has $h\left(\Delta_{q, d}\right)=h(q) \Delta_{q, d}$.

The group $G_{\text {analytic }} /\left(N \rtimes \mathbb{G}_{a}\right)=T^{+} \rtimes \widehat{\mathbb{Z}}$ is reductive, and therefore $N \rtimes \mathbb{G}_{a}$ is the unipotent radical of $G_{\text {analytic }}$. The affine group scheme $N \rtimes \mathbb{G}_{a}$ is topologically generated by $\left\{\exp \left(\Delta_{q, d}\right) \mid d\right.$ singular direction for $\left.q\right\} \cup\left\{\gamma_{u}\right\}$. The relations between these generators are unknown. The same holds for their logarithms $\left\{\Delta_{q, d}\right\} \cup\left\{\log \gamma_{u}\right\}$. For the group $N \rtimes \overline{\langle\gamma\rangle}$ with topological generators $\left\{\exp \left(\Delta_{q, d}\right)\right\} \cup\{\gamma\}$, one observes that the only relations are $\gamma \exp \left(\Delta_{q, d}\right) \gamma^{-1}=\exp \left(\Delta_{q, d-2 \pi}\right)$. A good translation in terms of generators of Lie algebras does not seem to exist.

Description of the analytic differential modules. As in [Van der Put and Singer 2003, Section 9.2], one can describe a differential module $M$ over $F$ by a structure on its solution space $V:=V(M)$, that is, $V(M)=\left(V,\left\{V_{q}\right\}, \gamma,\left\{S t_{d}\right\}\right)$. The maps $S t_{d}$ can be replaced by $s t_{d}=\log S t_{d}$, and $s t_{d}$ can be replaced by its components $s t_{q, d}$ (with $d$ a singular direction for $q$ ). These elements in $\operatorname{End}(V)$ map each $V_{q^{\prime}}$ to $V_{q+q^{\prime}}$ and so on. Thus $M$ is represented by a tuple $\left(V,\left\{V_{q}\right\}, \gamma,\left\{s t_{q, d}\right\}\right)$. 
Computation of $\boldsymbol{H}^{*}\left(\boldsymbol{G}_{\text {analytic }}, \boldsymbol{V}(\boldsymbol{M})\right)$. The computation uses the formula

$$
H^{*}\left(G_{\text {analytic }}, V(M)\right)=H^{*}\left(N \rtimes \mathbb{G}_{a}, V(M)\right)^{T^{+} \rtimes \widehat{\mathbb{Z}}} .
$$

First an example.

Example 5.1. Let $M=F e$, where $\partial(e)=-p e$ and $p=a_{k} z^{-k}+\cdots+a_{1} z^{-1}$ with $k \geq 1$ and $a_{k} \neq 0$. Then $M / \partial M \approx H^{1}\left(G_{\text {analytic }}, V(M)\right)$ has dimension $\operatorname{irr}(M)$; see below for the definition of the irregularity of $M$.

Proof. Let $\rho$ denote the action of $G_{\text {analytic }}$ on $V=V(M)=V_{p}=\mathbb{C} e(p) e$. Then $\rho$ is trivial for $N$ and $\gamma$. Further, for $h \in T=\operatorname{Hom}\left(2, \mathbb{C}^{*}\right)$, the map $\rho(h)$ is multiplication by $h(p)$.

Now $H^{1}\left(G_{\text {analytic }}, V\right)=H^{1}\left(N \rtimes \overline{\left\langle\gamma_{u}\right\rangle}, V\right)^{T \rtimes \overline{\left\langle\gamma_{s s}\right\rangle}}$. We may identify the cohomology group $H^{1}\left(N \rtimes \overline{\left\langle\gamma_{u}\right\rangle}, V\right)$ with the complex vector space of the algebraic homomorphism $f: N \rtimes \overline{\left\langle\gamma_{u}\right\rangle} \rightarrow V$ in such a way that there are only finitely many $q \in 2$ for which there is a $d$ with $f\left(\exp \left(\Delta_{q, d}\right)\right) \neq 0$.

Let $f$ be such a map, and suppose that $f$ is invariant under $T \rtimes \overline{\left\langle\gamma_{s s}\right\rangle}$. For $g \in T \rtimes \overline{\left\langle\gamma_{s s}\right\rangle}$ and $m \in N \rtimes \overline{\left\langle\gamma_{u}\right\rangle}$, one has $f(m)=(g f)(m)$. Since the latter is $\rho\left(g^{-1}\right) f\left(g m g^{-1}\right)$, one has $\rho(g) f(m)=f\left(g m g^{-1}\right)$. This applied with $g \in T$ and $m=\gamma_{u}$ or $m=\exp \left(\Delta_{q, d}\right)$ with $q \neq p$ yields $f\left(\gamma_{u}\right)=0$ and $f\left(\exp \left(\Delta_{q, d}\right)\right)=0$. Now apply this with $m=\gamma_{u} \exp \left(\Delta_{p, d}\right) \gamma_{u}^{-1}$ and $g=\gamma_{s s}$. Then $f(m)=f\left(\exp \left(\Delta_{p, d-2 \pi}\right)\right)$. Using that $f\left(\gamma_{u}\right)=0$ and $\rho\left(\gamma_{u}\right)=1$, one has $f(m)=f\left(\exp \left(\Delta_{p, d}\right)\right)$.

We then conclude that the invariant algebraic homomorphisms are described by $f\left(\gamma_{u}\right)=f\left(\exp \left(\Delta_{q, d}\right)\right)=0$ for $q \neq p$, and $f\left(\exp \left(\Delta_{p, d}\right)\right)=f\left(\exp \left(\Delta_{p, d-2 \pi}\right)\right)$ for all $d$. The dimension of the $H^{1}$ under consideration is therefore equal to the number of the singular directions $d$ modulo $2 \pi$ of $p$. This number is easily seen to be $k$. We note that this result coincides with the explicit calculations in [Van der Put and Singer 2003, Section 7.3].

B. Malgrange has introduced the irregularity of a differential module $M$ over $F$ as follows. Put $\widehat{M}:=\widehat{F} \otimes_{F} M$. The action of the operator $\partial$ on the exact sequence $0 \rightarrow M \rightarrow \widehat{M} \rightarrow \widehat{M} / M \rightarrow 0$ induces the long exact sequence

$0 \rightarrow \operatorname{ker}(\partial, M) \longrightarrow \operatorname{ker}(\partial, \widehat{M}) \longrightarrow \operatorname{ker}(\partial, \widehat{M} / M)$

$$
\longrightarrow M / \partial M \longrightarrow \widehat{M} / \partial \widehat{M} \longrightarrow \operatorname{coker}(\partial, \widehat{M} / M) \rightarrow 0 \text {. }
$$

His results are that $\operatorname{coker}(\partial, \widehat{M} / M)=0$, each term in this sequence has finite dimension, and $\operatorname{dim} \operatorname{ker}(\partial, \widehat{M})=\operatorname{dim} \widehat{M} / \partial \widehat{M}$ (see Corollary 4.1). The irregularity of $M, \operatorname{irr}(M)$, is defined as the dimension of $\operatorname{ker}(\partial, \widehat{M} / M)$. Using cohomology, we will reprove Malgrange's results and compute $\operatorname{irr}(M)$. 
Proposition 5.2 [Malgrange 1974]. Let $M$ be a differential module over $F$ with solution space $V(M)=\left(V,\left\{V_{q}\right\}, \gamma,\left\{s t_{q, d}\right\}\right)$. Then $\partial$ is surjective on $\widehat{M} / M$, and $\operatorname{irr}(M)=\sum_{q} \operatorname{deg}_{z^{-1}} q \cdot \operatorname{dim} V_{q}$.

Proof. First, we may identify the map $M / \partial M \rightarrow \widehat{M} / \partial \widehat{M}$ with the restriction map $R: H^{1}\left(N \rtimes G_{\text {formal }}, V\right) \rightarrow H^{1}\left(G_{\text {formal }}, V\right)$, where $V$ is the solution space of $M$. The five term exact sequence (with $G=G_{\text {analytic }}=N \rtimes G_{\text {formal }}$ ) reads

$0 \rightarrow H^{1}\left(G_{\text {formal }}, V^{N}\right) \longrightarrow H^{1}\left(G_{\text {analytic }}, V\right)$

$$
\longrightarrow H^{1}(N, V)^{G_{\text {formal }}} \longrightarrow H^{2}\left(G_{\text {formal }}, V^{N}\right) \longrightarrow \cdots .
$$

The term $H^{2}\left(G_{\text {formal }}, V^{N}\right)$ is zero. The description of $H^{1}(N, V)$ by 1 -cocycles modulo trivial 1-cocycles and the description of the pro-Lie algebra $\operatorname{Lie}(N)$ yield the exact sequence $0 \rightarrow V / V^{N} \rightarrow V^{\left(\left\{\Delta_{q, d}\right\}\right)} \rightarrow H^{1}(N, V) \rightarrow 0$. Taking invariants for the action of $G_{\text {formal }}$, one finds the exact sequence

$$
\begin{aligned}
0 \rightarrow & H^{0}\left(G_{\text {formal }}, V / V^{N}\right) \longrightarrow H^{0}\left(G_{\text {formal }}, V^{\left(\left\{\Delta_{q, d}\right\}\right)}\right) \longrightarrow \\
& H^{0}\left(G_{\text {formal }}, H^{1}(N, V)\right) \stackrel{\alpha_{1}}{\longrightarrow} H^{1}\left(G_{\text {formal }}, V / V^{N}\right) \longrightarrow H^{1}\left(G_{\text {formal }}, V^{\left(\left\{\Delta_{q, d}\right\}\right)}\right) .
\end{aligned}
$$

We will compute the terms of this exact sequence.

Claim. Irr $:=H^{0}\left(G_{\text {formal }}, V^{\left(\left\{\Delta_{q, d}\right\}\right)}\right)$ has dimension $\sum \operatorname{deg}_{z^{-1}} q \cdot \operatorname{dim} V_{q}$. Further $H^{1}\left(G_{\text {formal }}, V^{\left(\left\{\Delta_{q, d}\right\}\right)}\right)=0$, and so $\alpha_{1}$ is surjective.

Proof. $V^{\left(\left\{\Delta_{q, d}\right\}\right)}$ consists of the maps $f:\left\{\Delta_{q, d}\right\} \rightarrow V$ (with the property that only finitely many $q$ 's have a $d$ with $\left.f\left(\Delta_{q, d}\right) \neq 0\right)$. The action of $\gamma$ on $V^{\left(\left\{\Delta_{q, d}\right\}\right)}$ is given by $\left.(\gamma f)\left(\Delta_{q, d}\right)=\rho\left(\gamma^{-1}\right) f\left(\Delta_{\gamma(q), d-2 \pi}\right)\right)$. The map $f \mapsto(\gamma f)-f$ is seen to be surjective. It follows that $H^{1}\left(\overline{\langle\gamma\rangle}, V^{\left(\left\{\Delta_{q, d}\right\}\right)}\right)=0$ and that $H^{0}\left(\overline{\langle\gamma\rangle}, V^{\left(\left\{\Delta_{q, d}\right\}\right)}\right)$ consists of those maps satisfying $f\left(\Delta_{q, d}\right)=f\left(\Delta_{\gamma(q), d-2 \pi}\right)$. Taking now the invariants under $T$, one finds that $H^{1}\left(G_{\text {formal }}, V^{\left(\left\{\Delta_{q, d}\right\}\right)}\right)=0$ and $H^{0}\left(G_{\text {formal }}, V^{\left(\left\{\Delta_{q, d}\right\}\right)}\right)$ consists of the maps satisfying $f\left(\Delta_{q, d}\right) \in V_{q}$ and $f\left(\Delta_{q, d}\right)=f\left(\Delta_{\gamma(q), d-2 \pi}\right)$.

Write $V=V_{0} \oplus V_{q_{1}} \oplus \cdots \oplus V_{q_{r}}$, where $0, q_{1}, \ldots, q_{r}$ are distinct elements of 2 and the $V_{q_{i}} \neq 0$. We allow the possibility $V_{0}=0$. From the above it follows that Irr $:=H^{0}\left(G_{\text {formal }}, V^{\left(\left\{\Delta_{q, d}\right\}\right)}\right)$ has dimension $\sum_{i} \operatorname{deg}_{z^{-1}} q_{i} \cdot \operatorname{dim} V_{q_{i}}$.

Claim. The map $R: H^{1}\left(N \rtimes G_{\text {formal }}, V\right) \rightarrow H^{1}\left(G_{\text {formal }}, V\right)$ is surjective.

Proof. First we consider the long exact sequence

$$
\begin{aligned}
0 \rightarrow H^{0}\left(G_{\text {formal }}, V^{N}\right) & \longrightarrow H^{0}\left(G_{\text {formal }}, V\right) \longrightarrow H^{0}\left(G_{\text {formal }}, V / V^{N}\right) \\
& \stackrel{\alpha_{2}}{\longrightarrow} H^{1}\left(G_{\text {formal }}, V^{N}\right) \longrightarrow H^{1}\left(G_{\text {formal }}, V\right) \longrightarrow H^{1}\left(G_{\text {formal }}, V / V^{N}\right) \rightarrow 0 .
\end{aligned}
$$

We observe that $H^{0}\left(G_{\text {formal }}, V^{N}\right)=\operatorname{ker}(\partial, M)$ and that $H^{0}\left(G_{\text {formal }}, V\right)$ is equal to $\operatorname{ker}(\partial, \widehat{M})$. 
The morphism from the exact sequence

$$
0 \rightarrow H^{1}\left(G_{\text {formal }}, V^{N}\right) \longrightarrow H^{1}\left(G_{\text {analytic }}, V\right) \longrightarrow H^{0}\left(G_{\text {formal }}, H^{1}(N, V)\right) \rightarrow 0
$$

to the exact sequence

$$
0 \rightarrow H^{1}\left(G_{\text {formal }}, V^{N}\right) / \operatorname{im}\left(\alpha_{2}\right) \longrightarrow H^{1}\left(G_{\text {formal }}, V\right) \longrightarrow H^{1}\left(G_{\text {formal }}, V / V^{N}\right) \rightarrow 0
$$

is defined by the three maps $\alpha_{3}, R$ and $\alpha_{1}$, where $\alpha_{3}$ is induced by the identity map $H^{1}\left(G_{\text {formal }}, V^{N}\right) \rightarrow H^{1}\left(G_{\text {formal }}, V^{N}\right)$. The surjectivity of $\alpha_{1}$ implies that $R$ is surjective.

By the second claim, one has an exact sequence $0 \rightarrow \operatorname{im}\left(\alpha_{2}\right) \rightarrow \operatorname{ker}(R) \rightarrow$ $\operatorname{ker}\left(\alpha_{1}\right) \rightarrow 0$. Combining this with the results, observations and identifications of both claims, one finds Malgrange's exact sequence

$$
0 \rightarrow \operatorname{ker}(\partial, M) \longrightarrow \operatorname{ker}(\partial, \widehat{M}) \longrightarrow \operatorname{Irr} \longrightarrow M / \partial M \longrightarrow \widehat{M} / \partial \widehat{M} \rightarrow 0 .
$$

\section{Global differential equations}

Now $K$ is a finite extension of the differential field $\mathbb{C}(z)$. The universal $U(K)$ and $G(K, \partial)$ are far from known. For every place $v$, one has a canonical embedding $K \subset K_{v}$. We note that $K_{v}$ is isomorphic to $\mathbb{C}((t))$, where $t$ is a local parameter for $v$. The differentiation has the form $a \cdot \frac{d}{d t}$ for some nonzero element $a$ (which will not be of importance for our problems).

The embedding $K \subset K_{v}$ can be extended to an injection $U(K) \rightarrow U\left(K_{v}\right)$ of differential rings. This injection is not unique, but its image is. Thus the above arrow is unique up to a differential automorphism of $U(K) / K$, that is, an element of $G(K, \partial)$. For every place $v$, we make a choice for $U(K) \rightarrow U\left(K_{v}\right)$. This arrow induces an injective morphism $G\left(K_{v}, \partial\right) \rightarrow G(K, \partial)$ (which is unique up to conjugation by an element of $G(K, \partial))$.

Suppose $L \in K[\partial]$ is nonzero and $f \in K$. The equation $L(y)=f$ has a solution $y_{v} \in K_{v}$ at a place $v$ if $L$ is regular at $v$ and $f$ has no pole at $v$. Thus there exists a solution $y_{v}$ for all but finitely many places. Hence there is a well-defined map $K / L(K) \rightarrow \bigoplus_{v} K_{v} / L\left(K_{v}\right)$ of $\mathbb{C}$-vector spaces. The kernel of this map will be denoted by $\lg l(L)$. This vector space measures the failure of the local-global principle for differential equations.

For a differential module $M$ over $K$, one defines in a similar way the $\mathbb{C}$-vector space $\lg l(M):=\operatorname{ker} M / \partial M \rightarrow \bigoplus_{v}\left(K_{v} \otimes M\right) / \partial\left(K_{v} \otimes M\right)$.

Using the above and Section 2 one concludes that

$$
\lg l(M)=\operatorname{ker}\left(H^{1}(G(K, \partial), V(M)) \rightarrow \bigoplus_{v} H^{1}\left(G\left(K_{v}, \partial\right), V(M)\right)\right),
$$

and similarly for a differential operator $L$. 
Theorem 6.1. The $\mathbb{C}$-vector spaces $\operatorname{lgl}(L)$ and $\lg l(M)$ have finite dimension.

Proof. The statements for $L$ and $M$ are equivalent. We start with a differential module $M$ over $K$. As $K$ is a finite extension of $F:=\mathbb{C}(z)$, we may view $M$ as a differential module over $F$. The term $M / \partial M$ does not change. Consider a place $w$ of $F$ and the places $v_{1}, \ldots, v_{r}$ of $K$ above $w$. Then $F_{w} \otimes_{F} M$ can be identified with $\bigoplus_{i=1}^{r} K_{v_{i}} \otimes_{K} M$. This implies that $\lg l(M)$ does not change if one considers $M$ as differential module over $F$. Thus we may suppose that $K=\mathbb{C}(z)$.

Now we consider $L=a_{n} \partial^{n}+\cdots+a_{1} \partial+a_{0}$ with all $a_{i} \in K=\mathbb{C}[z]$ and $\operatorname{gcd}\left(a_{n}, \ldots, a_{0}\right)=1$. Consider an equation $L(y)=f$ with $f \in K$ that has a solution $y_{v} \in K_{v}$ for every place $v$. For each place $v \neq \infty$, we write this solution as $\left[y_{v}\right]+r_{v}$, where $\left[y_{v}\right]=\sum_{i \geq 1} c_{i} /(z-v)^{i}$ is the principal part of $y_{v}$. Only finitely many $\left[y_{v}\right]$ are nonzero, and $g:=f-\sum_{v \neq \infty} L\left(\left[y_{v}\right]\right)$ lies in $\mathbb{C}[z]$. Therefore it suffices to consider equations $L(y)=g$ with $g \in \mathbb{C}[z]$.

One observes that the operator $L: \mathbb{C}[z] \rightarrow \mathbb{C}[z]$ has a finite-dimensional cokernel. This implies that $\operatorname{lgl}(L)$ has finite dimension. More precisely, $\lg l(L)$ is the kernel of the obvious map $\mathbb{C}[z] / L(\mathbb{C}[z]) \rightarrow \bigoplus_{\text {all } v} \mathbb{C}(z)_{v} / L\left(\mathbb{C}(z)_{v}\right)$.

\section{Abelian differential equations}

As in Section 2, let $K$ be a differential field with an algebraically closed field of constants $C \neq K$ of characteristic 0 . A differential module $M$ (or an operator in $K[\partial])$ is called abelian if the differential Galois group $\operatorname{Gal}(M)$ is abelian. The corresponding Picard-Vessiot extension $L \supset K$ is also called abelian, and $\operatorname{Gal}(L / K)$ denotes its (differential) Galois group.

Any abelian linear algebraic group $G$ is a product of copies of $\mathbb{G}_{a}, \mathbb{G}_{m}$, and finite cyclic groups. By definition, the additive part $G^{+}$of $G$ is a product of copies of $\mathbb{G}_{a}$, and the multiplicative part $G^{\star}$ of $G$ is a product of copies of $\mathbb{G}_{m}$ and finite cyclic groups.

A linear algebraic group $G$ of additive type is described as $G=\operatorname{Spec}(C[W])$, where $W$ is a finite-dimensional $C$-vector space, $C[W]:=\bigoplus_{n \geq 0} \operatorname{sym}^{n} W$, and the comultiplication $m$ is given by $m(w)=(w \otimes 1)+(1 \otimes w)$ for all $w \in W$. A group scheme of additive type $G$ is by definition the projective limit of linear algebraic groups of additive type. It follows that it has also a description as $G=$ $\operatorname{Spec}(C[W])$ but now with $W$ any $C$-vector space. Clearly, the set $G(C)$ of the $C$-valued points of $G$ is identified with $\operatorname{Hom}_{C}(W, C)$. The set $\operatorname{Hom}\left(G, \mathbb{G}_{a}\right)$ of the morphisms can be identified with $W$ as follows. Fix a presentation $\mathbb{G}_{a}=C[t]$ with $m(t)=(t \otimes 1)+(1 \otimes t)$. Giving a morphism $G \rightarrow \mathbb{G}_{a}$ is equivalent to giving a $w \in W$ that is the image of $t$ for the map $C[t] \rightarrow C[W]$. By abuse of language, we will write $\operatorname{Hom}_{C}(W, C)$ for the group scheme $\operatorname{Spec}(C[W])$ of additive type. 
A linear algebraic group $G$ of multiplicative type can be described as $G=$ $\operatorname{Spec}(C[A])$, with $A$ a finitely generated abelian group, $C[A]$ the group ring of $A$ over $C$, and $m(a)=a \otimes a$ the comultiplication for all $a \in A$. A group scheme of multiplicative type is again defined as $G=\operatorname{Spec}(C[A])$, but now $A$ is an arbitrary abelian group. Now $G(C)=\operatorname{Hom}_{\mathbb{Z}}\left(A, C^{*}\right)$, and $\operatorname{Hom}\left(G, \mathbb{G}_{m}\right)=A$. By abuse of language, we will write $\operatorname{Hom}_{\mathbb{Z}}\left(A, C^{*}\right)$ for the group scheme $\operatorname{Spec}(C[A])$ of multiplicative type. Note that $\operatorname{Hom}\left(G_{1}, G_{2}\right)=0$ if $G_{1}$ is of additive type and $G_{2}$ is of multiplicative type or vice versa.

For any differential field $L$ one defines the $C$-vector subspace

$$
d(L):=\left\{f^{\prime} \mid f \in L\right\} \subset L
$$

and the subgroup $d \log (L):=\left\{f^{\prime} / f \mid f \in L^{*}\right\} \subset L$.

Theorem 7.1. Let $L$ be an abelian Picard-Vessiot extension of $K$. Then we have natural maps

$$
\alpha_{L / K}: \frac{K}{d \log (K)} \rightarrow \frac{L}{d \log (L)} \quad \text { and } \quad \beta_{L / K}: \frac{K}{d(K)} \rightarrow \frac{L}{d(L)} .
$$

induced by the inclusion $K \subseteq L$.

(i) $\operatorname{ker}\left(\alpha_{L / K}\right)$ is a $\mathbb{Z}$-module of finite type. $\operatorname{Hom}_{\mathbb{Z}}\left(\operatorname{ker}\left(\alpha_{L / K}\right), C^{*}\right)$ is considered as a linear algebraic group (of multiplicative type).

(ii) $\operatorname{ker}\left(\beta_{L / K}\right)$ is a finite-dimensional $C$-vector space. $\operatorname{Hom}_{C}\left(\operatorname{ker}\left(\beta_{L / K}\right), C\right)$ is considered as a linear algebraic group (of additive type).

(iii) There are canonical isomorphisms

$$
\begin{aligned}
& \psi_{L / K}^{\star}: \operatorname{Gal}(L / K)^{\star} \rightarrow \operatorname{Hom}_{\mathbb{Z}}\left(\operatorname{ker}\left(\alpha_{L / K}\right), C^{*}\right) \text { and } \\
& \psi_{L / K}^{+}: \operatorname{Gal}(L / K)^{+} \rightarrow \operatorname{Hom}_{C}\left(\operatorname{ker}\left(\beta_{L / K}\right), C\right)
\end{aligned}
$$

of linear algebraic groups.

The definitions of $\psi_{L / K}^{\star}$ and $\psi_{L / K}^{+}$are as follows. Let $\sigma \in \operatorname{Gal}(L / K)$. Take $\bar{f} \in \operatorname{ker}\left(\alpha_{L / K}\right)$ and $\bar{g} \in \operatorname{ker}\left(\beta_{L / K}\right)$ and images of $f \in K$ and $g \in K$. There are elements $F \in L^{*}$ and $G \in L$ such that $F^{\prime} / F=f$ and $G^{\prime}=g$. Then $\sigma(F)=c F$ for some $c \in C^{*}$, and $\sigma(G)=G+d$ for some $d \in C$. Then

$$
\psi_{L / K}^{\star}(\sigma)(\bar{f})=c \in C^{*} \quad \text { and } \quad \psi_{L / K}^{+}(\sigma)(\bar{g})=d \in C .
$$

It is easily verified that the above definitions do not depend on the choices of $f, F, g$ and $G$ and that the maps $\psi_{L / K}^{\star}$ and $\psi_{L / K}^{+}$are homomorphisms of algebraic groups. "Canonical" means that for abelian Picard-Vessiot extensions $K \subset L_{1} \subset L_{2}$, one has the obvious rules for composing the various $\psi^{\star}$ and $\psi^{+}$. 
Proof. The direct elementary proof that $\psi^{\star}$ and $\psi^{+}$are isomorphisms is somewhat long. We prefer to use [Van der Put and Reversat 2004, Lemma 1.1], which reduces the general case to the cases $\operatorname{Gal}(L / K) \in\left\{\mathbb{G}_{m}, C_{n}, \mathbb{G}_{a}\right\}$, where $C_{n}$ denotes the cyclic group of order $n>1$. For these cases we provide elementary proofs.

(i) $\operatorname{Gal}(L / K)=\mathbb{G}_{m}$. Then $L$ is the Picard-Vessiot extension of an equation $y^{\prime}=f y$ with $f \in K$ such that $y^{\prime}=m f y$ has, for every integer $m \geq 1$, only the trivial solution $y=0$ in $K$. Then $L$ is the transcendental extension $K(t)$ with $t^{\prime}=f t$, and $c \in \operatorname{Gal}(L / K)=C^{*}$ maps $t$ to $c t$.

The kernel of $\psi_{L / K}^{\star}: K / d \log (K) \rightarrow L / d \log (L)$ consists of the elements $h+$ $d \log (K)$ such that there exists $y \in L^{*}$ with $y^{\prime} / y=h$. The obvious elements $y \in L^{*}$ with $y^{\prime} / y \in K$ are $a t^{n}$ with $a \in K^{*}$ and $n \in \mathbb{Z}$. They produce $\mathbb{Z} \bar{f}$ in the kernel of $\psi_{L / K}^{\star}$. We want to show that there are no other elements $y \in L^{*}$ with $y^{\prime} / y \in K$. We may write $y=p(t) / q(t)$ with $p(t), q(t) \in K[t]$, where $p(t)$ and $q(t)$ are relatively prime, monic, and not divisible by $t$. Then

$$
\frac{y^{\prime}}{y}=\frac{p(t)^{\prime}}{p(t)}-\frac{q(t)^{\prime}}{q(t)} \in K
$$

implies that $p(t)^{\prime} / p(t)$ and $q(t)^{\prime} / q(t)$ both belong to $K$. Now $p(t)=t^{n}+\cdots+$ $p_{1} t+p_{0}$ for $p_{0} \neq 0$. Then $p(t)^{\prime}=p_{n-1}^{\prime} t^{n-1}+\cdots+p_{1}^{\prime} t+p_{0}^{\prime}+f\left(n t^{n}+\cdots+p_{1} t\right)=$ $a \cdot p(t)$ for some $a \in K$. This identity generates the equalities $a=n f$ and $p_{0}^{\prime}=a p_{0}$, and then $p_{0}^{\prime}=n f p_{0}$ contradicts the assumption on the equation $y^{\prime}=f y$ if $n>0$. Hence $p(t)=1$, and similarly $q(t)=1$. Thus we find that the kernel of $\psi_{L / K}^{\star}$ is $\mathbb{Z} \bar{f}$. The map $\operatorname{Gal}(L / K)=C^{*} \rightarrow \operatorname{Hom}\left(\mathbb{Z} \bar{f}, C^{*}\right)$ is obviously an isomorphism.

(ii) $\operatorname{Gal}(L / K)=C_{n}$. Then $L$ is the Picard-Vessiot extension of an equation $y^{\prime}=f y$ with $f \in K$ and $n$ minimal such that $y^{\prime}=n f y$ has a nonzero solution $y_{0} \in K$. Then $L=K(t)$ with $t^{n}=y_{0}$, and $\operatorname{Gal}(L / K)$ acts by multiplying $t$ by $n$-th roots of unity. The proof that the kernel of $\psi_{L / K}^{\star}$ is the cyclic group $\mathbb{Z} \bar{f}$ of order $n$ is similar to the one of case (i).

(iii) $\operatorname{Gal}(L / K)=\mathbb{G}_{a}$. Then $L=K(t) \neq K$, where $t$ satisfies a differential equation of the form $t^{\prime}=g$ with $g \in K$. An element $d \in \operatorname{Gal}(L / K)=\mathbb{G}_{a}=C$ maps $t$ to $t+d$. An element $h+d(K)$ lies in the kernel of $\psi_{L / K}^{+}$if and only if there exists an element $H \in L$ with $H^{\prime}=h$. The kernel clearly contains the $C$-subspace generated by $g+d(K)$. It suffices to show that the kernel contains no more elements. Now write $H=p(t) / q(t)+r(t)$ with relatively prime $p(t), q(t) \in K[t], q(t)$ monic, $\operatorname{deg} p(t)<\operatorname{deg} q(t)$, and $r(t) \in K[t]$. Suppose that

$$
H^{\prime}=r(t)^{\prime}+\frac{p(t)^{\prime} q(t)-p(t) q(t)^{\prime}}{q(t)^{2}}=h \in K .
$$

Then $p(t)^{\prime} q(t)-p(t) q(t)^{\prime}=\left(h-r(t)^{\prime}\right) q(t)^{2}$. If $q(t) \neq 1$, then, by comparing the degrees, one finds $r(t)^{\prime}=h$. The same holds if $q(t)=1$. We may write 
$r(t)=r_{d} t^{d}+\cdots+r_{1} t$, since the constant term of $r(t)$ is of no importance. Further $r_{d} \neq 0$. Then $r(t)^{\prime}=r_{d}^{\prime} t^{d}+\cdots+r_{1}^{\prime} t+g\left(r_{d} d t^{d-1}+\cdots+r_{1}\right)=h$. This implies $r_{d}^{\prime}=0$, and thus $r_{d}=c \in C^{*}$. For $d>1$, one finds the contradiction $r_{d-1}^{\prime}+g d c=0$. Hence $r(t)=c t$, and $h=c g$. This finishes the computation.

Theorem 7.1 admits the following corollary, which can be interpreted as an Artin correspondence for abelian Picard-Vessiot extensions.

Corollary 7.2. Let $K_{\mathrm{diff}}^{\mathrm{ab}}$ be the maximal abelian Picard-Vessiot extension of $K$. Then its Galois group $\operatorname{Gal}\left(K_{\mathrm{diff}}^{\mathrm{ab}} / K\right)$ satisfies the isomorphisms of affine group schemes over $C$ given by

$$
\begin{aligned}
& \psi_{K}^{\star}: \operatorname{Gal}\left(K_{\mathrm{diff}}^{\mathrm{ab}} / K\right)^{\star} \rightarrow \operatorname{Hom}_{\mathbb{Z}}\left(K / d \log (K), C^{*}\right) \text { and } \\
& \psi_{K}^{+}: \operatorname{Gal}\left(K_{\mathrm{diff}}^{\mathrm{ab}} / K\right)^{+} \rightarrow \operatorname{Hom}_{C}(K / d(K), C) .
\end{aligned}
$$

For $\psi_{K}^{\star} \times \psi_{K}^{+}$, write

$$
\psi_{K}: \operatorname{Gal}\left(K_{\mathrm{diff}}^{\mathrm{ab}} / K\right) \rightarrow \operatorname{Hom}_{\mathbb{Z}}\left(K / d \log (K), C^{*}\right) \times \operatorname{Hom}_{C}(K / d(K), C) .
$$

Then $\psi_{K}$ induces a bijection between the Picard-Vessiot extensions of finite type $K \subset L \subset K_{\mathrm{diff}}^{\mathrm{ab}}$ and the pairs $(Z, V) \subset(K / d \log (K), K / d(K))$, with $Z$ a subgroup of finite type and $V$ a $C$-vector subspace of finite dimension.

We note that $\psi_{K}^{\star}$ is the projective limit of the $\psi_{L / K}^{\star}$ that is taken over all PicardVessiot extensions of finite type $L \supset K$ contained in $K_{\text {diff }}^{\mathrm{ab}}$ (and similarly for $\psi_{K}^{+}$). The correspondence can be described as follows.

One associates to $(Z, V)$ the Picard-Vessiot field $L=\left(K_{\mathrm{diff}}^{\mathrm{ab}}\right)^{H}$, where $H$ is the kernel of the restriction map $\operatorname{Gal}\left(K_{\mathrm{diff}}^{\mathrm{ab}} / K\right) \rightarrow \operatorname{Hom}_{\mathbb{Z}}\left(Z, C^{*}\right) \times \operatorname{Hom}_{C}(V, C)$. This can be made even more explicit by giving $Z$ generators $\bar{a}_{1}, \ldots, \bar{a}_{r}$ over $\mathbb{Z}$ and by giving $V$ generators $\bar{b}_{1}, \ldots, \bar{b}_{s}$ over $C$. Then $L$ is the Picard-Vessiot extension for the set of equations

$$
y_{i}^{\prime}=a_{i} y_{i} \text { for } i=1, \ldots, r \text { and } z_{j}^{\prime}=b_{j} \text { for } j=1, \ldots, s \text {. }
$$

Conversely, $(\operatorname{ker}(K / d \log (K) \rightarrow L / d \log (L)), \operatorname{ker}(K / d(K) \rightarrow L / d(L)))$ is the pair associated to $L$.

We remark that the explicit presentation of the universal Picard-Vessiot ring and its automorphism group for the category of the abelian differential modules over $K$, as given in [Van der Put and Singer 2003, Chapter 10], also provides a proof for Theorem 7.1 and Corollary 7.2. 


\section{Computation of $\operatorname{lgl}(L)$ for abelian $L$}

We consider an abelian differential module $M$ over a finite extension $K$ of $\mathbb{C}(z)$ and compute the space $\operatorname{lgl}(M)$. It suffices to do this for an indecomposable $M$. This reduces the general case to the cases in which

(1) $M$ is of dimension 1 and trivial;

(2) $M$ has dimension 1 and differential Galois group $\mathbb{G}_{m}$;

(3) $M$ has dimension 1 and differential Galois group $C_{m}$ with $m>1$; and

(4) $M$ is of dimension $n$ and has differential Galois group $\mathbb{G}_{a}$.

8.1. The case of a trivial $L$. We consider a differential field $K$ that is a finite extension of $\mathbb{C}(z)$, and let $L=d / d z$. The solution space $V=\mathbb{C} 1$, and $\lg l(L)$ is the kernel of the map $H^{1}(G(K, \partial), V) \rightarrow \bigoplus_{v} H^{1}\left(G\left(K_{v}, \partial\right), V\right)$. Since the groups $G_{*}$ act trivially on $V$, the term $H^{1}$ coincides with the morphism $G_{*} \rightarrow \mathbb{G}_{a}$. A morphism factorizes over the additive factor of the abelianized group $\left(G_{*}\right)_{\mathrm{ab}}^{+}$. For the group $G(K, \partial)$, the additive factor (see Corollary 7.2) can be written as $\operatorname{Hom}_{C}(\Omega(K) / d K, C)$, where $\Omega(K)$ is the differential module for $K / \mathbb{C}$. Further $\operatorname{Hom}\left((G(K, \partial))_{\mathrm{ab}}^{+}, \mathbb{G}_{a}\right)$ identifies with $\Omega(K) / d K$. Similar statements hold for the groups $G\left(K_{v}, \partial\right)$.

Let $X$ be the curve associated to $K$. The points $x$ of $X$ correspond to the $v$ 's. Further $\Omega(K)$ identifies with the space of all meromorphic differential forms on $X$. From [Van der Put and Reversat 2004, Section 1.3], we recall the exact sequence

$$
0 \rightarrow H_{\mathrm{DR}}^{1}(X, \mathbb{C}) \longrightarrow \Omega(K) / d K \longrightarrow \bigoplus_{x \in X} \Omega(x) / d\left(K_{x}\right) \longrightarrow \mathbb{C} \rightarrow 0 .
$$

This implies the following result.

Proposition 8.2. For the finite field extension $K \supset \mathbb{C}(z)$ associated to a curve $X$ over $\mathbb{C}$ of genus $g, \operatorname{lgl}(\mathrm{d} / \mathrm{dz})$ is canonically isomorphic to the $2 \mathrm{~g}$-dimensional vector space $H_{\mathrm{DR}}^{1}(X, \mathbb{C})$.

8.3. The $\mathbb{G}_{\boldsymbol{m}}$ case. $K$ is again a finite extension of $\mathbb{C}(z)$, which corresponds to a curve $X$ over $\mathbb{C}$. Let the point $p \in X$ have local analytic parameter $t$. As before, one writes $K_{p}$ for the completion of $K$ at the valuation induced by $p$. Thus $K_{p}=\mathbb{C}((t))$. The subfield of the convergent Laurent series $\mathbb{C}(\{t\})$ is denoted by $K_{p}^{\text {an }}$. Further $\Omega\left(K_{p}\right):=\mathbb{C}((t)) d t$ and $\Omega\left(K_{p}^{\mathrm{an}}\right):=\mathbb{C}(\{t\}) d t$.

The operator $L$ has the form $\frac{d}{d z}-f$ with $f \in K^{*}$ such that $d y=m \cdot y f d z$ has, for any integer $m \geq 1$, only the trivial solution $y=0$ in $K$. One considers the map, again called $L$, given by $y \in K \mapsto d y-y f d z \in \Omega(K)$. Let $H \subset \Omega(K)$ be the subspace consisting of the elements $\omega$ such that there exists for every point $p$ a formal local solution $y \in K_{p}$ of $L(y)=\omega$. Then $\lg l(L)$ is the cokernel of $L: K \rightarrow H$. 
A point $p$ is regular for $L$ if there exists a nonzero $g \in K_{p}$ with $L(g)=0$. In fact, this $g$ lies in $K_{p}^{\text {an }}$. Further $p$ is regular if and only if $f d z$ has at most a pole of order 1 at $p$ and $\operatorname{Res}_{p}(f d z) \in \mathbb{Z}$. For a regular point $p$ of the map $L: K_{p} \rightarrow \Omega\left(K_{p}\right)$, the kernel and cokernel have dimension 1. The same holds for $L: K_{p}^{\text {an }} \rightarrow \Omega\left(K_{p}^{\mathrm{an}}\right)$. This is seen be writing $y=g h$ with $h \in K_{p}$. Then $L(y)=L(g h)=g d h$.The kernel of $L$ is $\mathbb{C} g$, and $\omega$ lies in the image of $L$ if and only if $\operatorname{Res}_{p}\left(g^{-1} \omega\right)=0$.

Further, a point $p$ is regular singular if $f d z$ has a pole of order 1 at $p$ and if $\operatorname{Res}_{p}(f d z) \notin \mathbb{Z}$. In this case the map $L: K_{p} \rightarrow \Omega\left(K_{p}\right)$ is bijective and the same holds for $L: K_{p}^{\text {an }} \rightarrow \Omega\left(K_{p}^{\text {an }}\right)$.

A point $p$ is irregular singular if $f d z$ has a pole of order $d+1$ with $d>0$. The integer $d$ is the irregularity $\operatorname{irr}_{p}$ of $p$. In this case, the map $L: K_{p} \rightarrow \Omega\left(K_{p}\right)$ is bijective. Further $L: K_{p}^{\text {an }} \rightarrow \Omega\left(K_{p}^{\text {an }}\right)$ is injective and its cokernel $\operatorname{Irr}(p, L)$ has dimension $d=\operatorname{irr}_{p}$.

Let $\operatorname{Sol}(L)$ denote the subsheaf of the sheaf of meromorphic functions $M$ on $X$, defined by $\operatorname{Sol}(L)(U)=\{y \in M(U) \mid L(y)=0\}$ for any open $U \subset X$. It is a sheaf of $\mathbb{C}$-vector spaces. By assumption $H^{0}(X, \operatorname{Sol}(L))=0$.

Lemma 8.4. $\operatorname{dim} H^{1}(X, \operatorname{Sol}(L))=2 g-2+\# S$ and $H^{2}(X, \operatorname{Sol}(L))=0$.

Proof. Let $S \subset X$ denote the set of singular points of $L$.

First we suppose $S \neq \varnothing$. The restriction of the sheaf $\mathscr{L}=\operatorname{Sol}(L)$ to $X^{*}:=X \backslash S$ is locally isomorphic to the constant sheaf $\underline{\mathbb{C}}$. It is given by a nontrivial homomorphism of the fundamental group $\pi_{1}:=\pi_{1}\left(X^{*}\right) \rightarrow \operatorname{GL}(V)$, where $V$ is a 1-dimensional vector space. Then $H^{i}\left(X^{*}, \mathscr{L}\right)$ equals the cohomology group $H^{i}\left(\pi_{1}, V\right)$ for all $i$. The group $\pi_{1}$ is free on $r:=2 g-1+\# S$ generators. Let $t_{1}, \ldots, t_{r}$ denote these free generators. The action of $t_{i}$ on $V$ is multiplication by some $\alpha_{i} \in \mathbb{C}^{*}$. The cohomology groups $H^{*}\left(\pi_{1}, V\right)$ are the cohomology groups of the complex $0 \rightarrow V \rightarrow V^{r} \rightarrow 0$, where the nontrivial arrow is defined by $v \mapsto\left(t_{i} v-v\right)_{i=1, \ldots, r}$. Since some $\alpha_{i}$ is not equal to 1 , one has $H^{i}\left(\pi_{1}, V\right)=0$ for $i=0,2$, and $\operatorname{dim} H^{1}\left(\pi_{1}, V\right)=r-1$.

We claim that $H^{1}(X, \mathscr{L}) \rightarrow H^{1}\left(X^{*}, \mathscr{L}\right)$ is an isomorphism. Let $U$ be the disjoint union of small disks around the points of $S$, and let $U^{*}=U \backslash S$. The Mayer-Vietoris sequence for the covering $\left\{X^{*}, U\right\}$ yields the exact sequence

$$
0 \rightarrow H^{1}(X, \mathscr{L}) \longrightarrow H^{1}\left(X^{*}, \mathscr{L}\right) \oplus H^{1}(U, \mathscr{L}) \longrightarrow H^{1}\left(U^{*}, \mathscr{L}\right) \longrightarrow \cdots
$$

Consider a small disk $D_{p}$ around a point $p \in S$, and let $D_{p}^{*}=D_{p} \backslash\{p\}$. We can identify $D_{p}$ with $D:=\{z \in \mathbb{C}|| z \mid<1\}$ and $D_{p}^{*}$ with $D^{*}:=\{z \in \mathbb{C}|0<| z \mid<1\}$; we identify restriction of $\mathscr{L}$ with the kernel of the morphism $M: O_{D} \rightarrow O_{D}$ (here $O_{D}$ denotes the sheaf of the holomorphic functions on $D$ ), given by $y \mapsto z y^{\prime}-a y$ with any $a \in \mathbb{C} \backslash \mathbb{Z}$. The sequence $0 \rightarrow \operatorname{ker} M \rightarrow O_{D} \rightarrow O_{D} \rightarrow 0$ is exact. One verifies that $M: H^{0}\left(E, O_{D}\right) \rightarrow H^{0}\left(E, O_{D}\right)$ is bijective for $E=D$ and $E=D^{*}$. 
Further $H^{i}\left(E, O_{D}\right)=0$ for $E=D, E=D^{*}$ and $i=1,2$. This implies that $H^{i}(E, \operatorname{ker} M)=0$ for $E=D, E=D^{*}$ and $i=1,2$. In this way we have verified that $H^{1}(U, \mathscr{L})=H^{1}\left(U^{*}, \mathscr{L}\right)=0$ and this proves the claim. Moreover $H^{2}(X, \mathscr{L})=0$.

Now we consider the case $S=\varnothing$. The sheaf $\mathscr{L}=\operatorname{Sol}(L)$ is locally isomorphic to the constant sheaf $\mathbb{C}$. However, $\mathscr{L}$ is not equal to the constant sheaf since we have supposed that the equation $L(y)=0$ has only the solution $y=0$ in $K$. In particular, the genus $g$ of $X$ is $\geq 1$. Take any point $p \in X$. Then $H^{1}(X \backslash\{p\}, \mathscr{L})$ is isomorphic to $H^{1}(\pi, V)$, where $\pi$ is the fundamental group of $X^{*}:=X \backslash\{p\}$, free on $2 g$ generators and $V$ is the 1-dimensional vector space such that the restriction of $\mathscr{L}$ to $X \backslash\{p\}$ corresponds to a nontrivial action of $\pi$ on $V$. Thus $\operatorname{dim} H^{1}(\pi, V)=2 g-1$. Let $D$ be a small disk around $p$, and put $D^{*}=D \backslash\{p\}$. The Mayor-Vietoris sequence for the covering $\left\{X^{*}, D\right\}$ of $X$ yields an exact sequence

$0 \rightarrow H^{1}(X, \mathscr{L}) \longrightarrow H^{1}\left(X^{*}, \mathscr{L}\right) \oplus H^{1}(D, \mathscr{L}) \longrightarrow H^{1}\left(D^{*}, \mathscr{L}\right) \longrightarrow H^{2}(X, \mathscr{L}) \rightarrow 0$.

Now $H^{1}(D, \mathscr{L})=0$, and $H^{1}\left(D^{*}, \mathscr{L}\right)$ has dimension 1 . According to Lemma 8.5 , the space $H^{2}(X, \mathscr{L})$ is dual to $H^{0}\left(X, \mathscr{L}^{*}\right)$ and therefore 0 . It follows that $\operatorname{dim} H^{1}(X, \mathscr{L})=2 g-2$.

The following result is cited in [Katz 1990, Section 2.9.8.2, p. 68]. Though a proof can be deduced from [Deligne 1970, Proposition II.6.20], ${ }^{1}$ we give here a direct proof.

Lemma 8.5. Let $\mathcal{M}$ be a sheaf of $\mathbb{C}$-vector spaces on $X$ locally isomorphic to the constant sheaf $\underline{\mathbb{C}}^{k}$. Then $\sum_{i=0}^{2}(-1)^{i} \operatorname{dim} H^{i}(X, \mu)=k(2-2 g)$. Moreover, there is a canonical isomorphism $H^{2}(X, \mathcal{M}) \rightarrow H^{0}\left(X, M^{*}\right)^{*}$.

Proof. The definition of the dual $\mu^{*}$ is rather obvious. Consider the exact sequence $0 \rightarrow M \rightarrow M \otimes_{\mathbb{C}} O_{X} \rightarrow M \otimes_{\mathbb{C}} \Omega(X) \rightarrow 0$ obtained by tensoring the exact sequence $0 \rightarrow \underline{\mathbb{C}} \rightarrow O_{X} \rightarrow \Omega(X) \rightarrow \overline{0}$ with $\mu$ over $\underline{\mathbb{C}}$.

This induces an exact sequence

$$
0 \rightarrow H^{0}(\mathcal{M}) \cdots H^{1}\left(\mathcal{M} \otimes O_{X}\right) \stackrel{A}{\longrightarrow} H^{1}(\mathcal{M} \otimes \Omega(X)) \longrightarrow H^{2}(\mathcal{M}) \rightarrow 0
$$

of cohomology groups above $X$. The vector bundle $M \otimes O_{X}$ has rank $k$. Its degree is zero since the line bundle $\Lambda^{k}\left(M \otimes O_{X}\right)$ admits a connection without singularities. This implies the formula. By Serre duality, $A$ is the dual of

$$
H^{0}\left(\mathcal{M}^{*} \otimes O_{X}\right) \stackrel{B}{\longrightarrow} H^{0}\left(\mathcal{M}^{*} \otimes \Omega(X)\right) .
$$

Now $(\operatorname{ker} B)^{*} \cong \operatorname{coker} A$ yields the required duality $H^{2}(\mathcal{M})^{*} \cong H^{0}\left(\mathcal{M}^{*}\right)$.

\footnotetext{
${ }^{1}$ We thank the referee for pointing out these two references.
} 
One defines the skyscraper sheaf 2 on $X$ by the exact sequence of sheaves

$$
0 \rightarrow \operatorname{Sol}(L) \longrightarrow M \stackrel{L}{\longrightarrow} \Omega_{\mathrm{mer}} \longrightarrow 2 \rightarrow 0,
$$

where $\Omega_{\text {mer }}$ denote the sheaf of the meromorphic differential forms on $X$. The sheaf 2 is the sheaf $\bigoplus_{p \in X} E_{p}$. Each term $E_{p}$ denotes a sheaf whose stalk is zero at the points $q \neq p$ and whose stalk at $p$ is the finite-dimensional vector space $\Omega\left(K_{p}^{\mathrm{an}}\right) / L\left(K_{p}^{\mathrm{an}}\right)$. By definition, a section of 2 above an open set $U$ is an element of $\prod_{p \in U} \Omega\left(K_{p}^{\mathrm{an}}\right) / L\left(K_{p}^{\mathrm{an}}\right)$ that has discrete support.

Let the sheaf $\mathscr{H}$ denote the image of $L$. The exact sequences

$$
0 \rightarrow \operatorname{Sol}(L) \longrightarrow M \longrightarrow \mathscr{H} \rightarrow 0 \text { and } 0 \rightarrow \mathscr{H} \longrightarrow \Omega_{\mathrm{mer}} \longrightarrow 2 \rightarrow 0
$$

induce long exact sequences for their cohomology on $X$. In combination with Lemma 8.4, the first one yields $H^{i}(X, \mathscr{H})=0$ for $i=1,2$ and also the exact sequence $0 \rightarrow K \rightarrow H^{0}(X, \mathscr{H}) \rightarrow H^{1}(X, \operatorname{Sol}(L)) \rightarrow 0$.

The second one yields the exact sequence

$$
0 \rightarrow H^{0}(X, \mathscr{H}) \longrightarrow \Omega(K) \longrightarrow \bigoplus_{p \in X} \Omega\left(K_{p}^{\mathrm{an}}\right) / L\left(K_{p}^{\mathrm{an}}\right) \rightarrow 0 .
$$

The space $H_{0}:=H^{0}(X, \mathscr{H})$ consists of the differential forms $\omega \in \Omega(K)$ such that at every point $p$ there exists an element $y \in K_{p}^{\text {an }}$ with $L(y)=\omega$. Let $H \subset \Omega(K)$ denote the subspace of the $\omega$ such that for every point $p$ there exists a $y \in K_{p}$ with $L(y)=\omega$. We recall that $\lg l(L)$ is the cokernel of $L: K \rightarrow H$.

The last exact sequence implies that $R: \Omega(K) \rightarrow \bigoplus_{p \in \operatorname{Irr}} \operatorname{Irr}(p, L)$ is surjective. Here Irr denotes the set of the irregular singular points and we recall that $\operatorname{Irr}(p, L)=$ $\Omega\left(K_{p}^{\mathrm{an}}\right) / L\left(K_{p}^{\mathrm{an}}\right)$. It follows that the dimension of $\lg l(L)$ is the sum $\sum_{p \in \operatorname{Irr}} \operatorname{irr}_{p}$, and the dimension of the cokernel of $L: K \rightarrow H_{0}$. The latter can be identified with $H^{1}(X, \operatorname{Sol}(L))$. Thus we have proved the following.

Theorem 8.6. $\operatorname{dim} \operatorname{lgl}(L)=2 g-2+\# S+\sum_{p \in \operatorname{Irr}} \operatorname{irr}_{p}$.

Example 8.7. Consider the regular singular, first order operator on $\mathbb{P}^{1}$ given by

$$
L=\frac{d}{d z}+\sum_{j=1}^{r} \frac{\lambda_{j}}{z-p_{j}} \quad \text { with } \lambda_{j} \notin \mathbb{Z} \text { for all } j .
$$

$S=\left\{p_{1}, \ldots, p_{r}\right\}$ if $\sum \lambda_{j} \in \mathbb{Z}$, and otherwise $S=\left\{p_{1}, \ldots, p_{r}, \infty\right\}$. According to Theorem 8.6, $\operatorname{dim} \operatorname{lgl}(L)$ is $r-2$ in the first case and $r-1$ in the second one.

An explicit calculation of $\operatorname{lgl}(L)$ using the proof of Theorem 6.1: $L$ is replaced by the operator

$$
L^{+}=\prod_{j=1}^{r}\left(z-p_{j}\right) L=\prod_{j=1}^{r}\left(z-p_{j}\right) \frac{d}{d z}+q(z) .
$$


Then $\operatorname{lgl}(L)=\operatorname{lgl}\left(L^{+}\right)$, and the latter is equal to the kernel of

$$
\mathbb{C}[z] / L^{+}(\mathbb{C}[z]) \rightarrow \bigoplus_{p \in \mathbb{P}^{1}} \mathbb{C}(z)_{p} / L^{+}\left(\mathbb{C}(z)_{p}\right) .
$$

The map $\mathbb{C}[z] / L^{+}(\mathbb{C}[z]) \rightarrow \mathbb{C}(z)_{p} / L\left(\mathbb{C}(z)_{p}\right)$ is the zero map except when $p=\infty$ and $\sum \lambda_{j} \in \mathbb{Z}$. In verifying that $\operatorname{dim} \operatorname{coker}\left(L^{+}, \mathbb{C}[z]\right)=r-1$, one has to consider separately three cases: $\sum \lambda_{j}=-n$ with $n>0$ an integer, $\sum \lambda_{j}=0$, and $\sum \lambda_{j}$ is not an integer $\leq 0$.

Example 8.8. The irregular singular operator $L=d / d z-z^{5}$ on $\mathbb{P}^{1}$ satisfies $\operatorname{dim} \operatorname{lgl}(L)=5$. Indeed, $g=0, S=\{\infty\}$ and $\operatorname{irr}_{\infty}=5$. The latter is verified by writing a multiple of $L$ in terms of the parameter $t=z^{-1}$ as $d / d t+t^{6}$.

The explicit method of Theorem 6.1 and the observation that $L$ is bijective on $\mathbb{C}(z)_{\infty}$ yield that $\operatorname{lgl}(L)$ is equal to the cokernel of $L$ on $\mathbb{C}[z]$. The latter has as basis (the images of) $1, z, z^{2}, z^{3}, z^{4}$.

Example 8.9. $L=z^{5} \frac{d}{d z}+1+a z^{4}$ with $a \notin \mathbb{Z}$ acting on $\mathbb{P}^{1}$ has $\operatorname{dim} \lg l(L)=4$. Indeed, $g=0, S=\{0, \infty\}$, and $\operatorname{irr}_{0}=4$. Now the method of Theorem 6.1: The cokernel of $L$ on $\mathbb{C}[z]$ has a basis of representatives $\left\{1, z, z^{2}, z^{3}\right\}$. The equation $L y=f$ with $f$ a polynomial of degree $\leq 3$ has a local solution at $\infty$. Hence $\operatorname{dim} \operatorname{lgl}(L)=4$.

Example 8.10. Consider $K=\mathbb{C}(z)[y]$ with $y^{2}=\left(z-a_{1}\right) \cdots\left(z-a_{2 g+2}\right)$. The trivial differential module $K$ with $\partial 1=0$ satisfies $\operatorname{dim} \lg l(K)=2 g$. This follows at once from Proposition 8.2. Another way to calculate $\operatorname{lgl}(K)$ is to view $K$ as the two-dimensional differential module $\mathbb{C}(z) 1 \oplus \mathbb{C}(z) y$ with $\partial 1=0$ and $\partial y=$ $\left(\sum_{j=1}^{2 g+2}(1 / 2) /\left(z-a_{j}\right)\right) y$. The first factor has $l g l=0$, and for the second factor, Theorem 8.6 yields $\operatorname{dim} l g l=2 g$.

Remark 8.11. The referee has noted that the formulas obtained in this section (and the next one) resemble known results like [Katz 1990, Theorem 2.9.9 and its corollaries]. At present we have not found any relation.

8.12. The $\boldsymbol{C}_{\boldsymbol{m}}$ case with $\boldsymbol{m}>\mathbf{1}$. This case is rather similar to the case $\mathbb{G}_{m}$. The only new point is that there are only regular singularities, since the differential Galois group is finite. With the same notations as above, the result is this:

Proposition 8.13. $\operatorname{dim} \lg l(L)=2 g-2+\# S$.

8.14. The $\mathbb{G}_{\boldsymbol{a}}$ case. The action of $\mathbb{G}_{a}$ on the solution space is given by $t \mapsto e^{t} N$, where $N$ is a nilpotent $n \times n$ matrix consisting of one Jordan block. Using that $K$ is a $C_{1}$-field and that the differential Galois group is connected, one finds a matrix differential equation for $M$ of the form $d / d z-f N$ for some nonzero $f \in K$; see [Van der Put and Singer 2003, Corollary 1.32]. We note that this differential 
equation is regular singular. The singular points $p \in X$, where $X$ denotes the curve associated to $K$, are precisely the points where the residue of $f d z$ is not zero. Let $S \subset X$ denote the set of the singular points. If $S$ is not empty, then $S$ contains at least 2 points since the sum of the residues of $f d z$ is zero. If $S=\varnothing$, then $f d z$ could be exact. In that case we are in the situation of Section 8.1, and therefore we assume that $f d z$ is not exact.

We want to compare the cokernel of $d / d z-f N$ acting upon $K^{n}$ with the cokernels of the same operator acting upon all $K_{p}^{n}$ for $p \in X$.

Computation for $\boldsymbol{n}=\mathbf{2}$. We consider the operator

$$
\begin{aligned}
& L\left(\left(\begin{array}{l}
y_{1} \\
y_{2}
\end{array}\right)\right):=\left(\frac{d}{d z}-f N\right)\left(\begin{array}{l}
y_{1} \\
y_{2}
\end{array}\right)=\left(\begin{array}{c}
y_{1}^{\prime}-f y_{2} \\
y_{2}^{\prime}
\end{array}\right): K^{2} \rightarrow K^{2}, \text { or equivalently } \\
& L\left(\left(y_{1}, y_{2}\right)=\left(d y_{1}-f y_{2} d z, d y_{2}\right): K^{2} \rightarrow \Omega(K)^{2} .\right.
\end{aligned}
$$

Let $H \subset \Omega(K)^{2}$ be the complex subspace consisting of the elements $\left(\omega_{1}, \omega_{2}\right)$ such that there is a formal local solution at each point $v$ of $\mathbb{P}^{1}$. Then $\operatorname{lgl}(d / d z-f N)$ is the cokernel of the map $L: K^{2} \rightarrow H$.

We recall that $X$ is the curve associated to $K$. Consider the $\mathbb{C}$-linear map $R: H \rightarrow H_{\mathrm{DR}}^{1}(X, \mathbb{C})$ given by $\left(\omega_{1}, \omega_{2}\right) \mapsto \overline{\omega_{2}}$, where $\overline{\omega_{2}}$ is the image of $\omega_{2}$ in $H_{\mathrm{DR}}^{1}(X, \mathbb{C})$. This is well defined since all the residues of $\omega_{2}$ are 0 . Now we investigate the image of $R$.

Suppose that $S$ is not empty. Take an $\omega_{2}$ (with all residues 0 ) representing a given element in $H_{\mathrm{DR}}^{1}(X, \mathbb{C})$. We have to produce an $\omega_{1}$ such that $\left(\omega_{1}, \omega_{2}\right) \in H$. We will use the existence of a meromorphic differential form $\omega$ for any prescription of its residues $\operatorname{Res}_{p}(\omega)=a_{p}$ such that almost all $a_{p}$ are 0 and $\sum a_{p}=0$.

For any point $p \notin S$ such that neither $\omega_{1}$ nor $f d z$ have a pole at $p$, we take $a_{p}=0$. For a point $p \notin S$ such that $\omega_{1}$ has a pole at $p$ or $f d z$ has a pole at $p$, we define $a_{p}$ by $a_{p}+\operatorname{Res}_{p}\left(y_{2} f d z\right)=0$ (where $d y_{2}=\omega_{1}$ locally at $p$ ). For a singular point $p \in S$, we take a local solution $y_{2}$ of $d y_{2}=\omega_{2}$. Then, since we may change $y_{2}$ into $y_{2}+c$ for any $c \in \mathbb{C}$, we have that for any choice of $a_{p}$, there is a constant $c$ such that the residue of $a_{p}+\operatorname{Res}_{p}\left(y_{2} f d z+c f d z\right)$ equals 0 . Since $S$ is not empty, we can choose the last values of $a_{p}$ such that $\sum a_{p}=0$. Any $\omega_{1}$ with these residues satisfies $\left(\omega_{1}, \omega_{2}\right) \in H$. Thus $R$ is surjective.

Consider the map $L: K^{2} \rightarrow H_{0}:=\left\{\left(\omega_{1}, \omega_{2}\right) \in H \mid \overline{\omega_{2}}=0\right\}$. Dividing $H_{0}$ by $L\left(\left\{\left(0, y_{2}\right) \mid y_{2} \in K\right\}\right)$, we have to compute the cokernel of $L: K \oplus \mathbb{C} \rightarrow H_{00}$ with $H_{00}:=\left\{\left(\omega_{1}, \omega_{2}\right) \in H \mid \omega_{2}=0\right\}$. Any $\left(\omega_{1}, 0\right) \in H_{00}$ is mapped to the image of $\left(\operatorname{Res}_{v}\left(\omega_{1}\right)\right)_{v \in S}$ in the space

$$
\left\{\left(a_{v}\right)_{v \in S} \in \mathbb{C}^{S} \mid \sum a_{v}=0\right\} / \mathbb{C}\left(\operatorname{Res}_{v}(f d z)\right)_{v \in S}
$$


This map is surjective. The kernel of this map consists of the $\omega_{1}$ for which there exists a constant $c$ such that every residue of $\omega_{1}+c f d z$ is zero (we note that $c$ is unique). This leads to the statement that the cokernel of $L: K \oplus \mathbb{C} \rightarrow H_{00}$ has dimension $(-2+\# S)+\operatorname{dim} H_{\mathrm{DR}}^{1}(X, \mathbb{C})$, and the cokernel of $L: K^{2} \rightarrow H$ has dimension $2 g+(-2+\# S)+2 g$.

Suppose that $S=\varnothing$. Let $M$ and $\Omega_{\text {mer }}$ be the sheaves on $X$ (for the ordinary complex topology) of the meromorphic functions and the meromorphic differential forms. Both sheaves have $H^{i}=0$ for $i \geq 1$. Consider the morphism $L: M^{2} \rightarrow \Omega_{\mathrm{mer}}^{2}$ defined by $\left(y_{1}, y_{2}\right) \mapsto\left(d y_{1}-y_{2} f d z, d y_{2}\right)$. Let $\mathcal{H} \subset \Omega_{\text {mer }}^{2}$ be the image of $L$. Then $H^{0}(X, \mathscr{H})$ consists of the pairs $\left(\omega_{1}, \omega_{2}\right) \in \Omega(K)^{2}$ such that the equation $\left(d y_{1}-y_{2} f d z, d y_{2}\right)=\left(\omega_{1}, \omega_{2}\right)$ has a local solution everywhere. The sheaf $\mathscr{L}$ is defined by the exact sequence $0 \rightarrow \mathscr{L} \rightarrow M^{2} \rightarrow \mathscr{H} \rightarrow 0$. The sheaf $\mathscr{L}$ is locally isomorphic to the constant sheaf $\mathbb{C}^{2}$. Taking the cohomology above $X$ one finds the exact sequence $0 \rightarrow H^{0}(X, \mathscr{L}) \rightarrow K^{2} \rightarrow H^{0}(X, \mathscr{H}) \rightarrow H^{1}(\mathscr{L}) \rightarrow 0$. Thus $H^{1}(\mathscr{L})$ identifies with $\lg l(d / d z-f N)$. It is easily seen that $H^{0}(\mathscr{L})$ and $H^{0}\left(\mathscr{L}^{*}\right)$ have both dimension 1. Lemma 8.5 implies that $\operatorname{dim} H^{1}(\mathscr{L})=4 g-2$.

Proposition 8.15. Suppose that $f d z$ is not exact and that $n=2$. Then the dimension of $\operatorname{lgl}(d / d z-f N)$ is $4 g-2+\# S$, where $S$ consists of the singular points of $d / d z-f N$, that is, the points $p$ with $\operatorname{Res}_{p}(f d z) \neq 0$.

Theorem 8.16. Suppose that $f d z$ is not exact. Consider $L=(d / d z-f N)$, where $N \in \operatorname{End}\left(\mathbb{C}^{n}\right)$ is a nilpotent matrix with one Jordan block. Let $S$ be the set of singular points of $L$, that is, the points $p$ with $\operatorname{Res}_{p}(f d z) \neq 0$. Then $\lg l(L)$ has dimension $2 g+(n-1) \cdot(2 g-2+\# S)$.

Proof. For $S=\varnothing$ one easily verifies that the above method, explained for $n=2$, holds for any $n \geq 2$. For $S \neq \varnothing$ the proof uses induction with respect to $n$. For notational convenience we only consider $n=3$.

$\operatorname{lgl}(L)$ is the cokernel of the map $L: K^{3} \rightarrow H \subset \Omega(K)^{3}$ given by

$$
L\left(y_{1}, y_{2}, y_{3}\right)=\left(d y_{1}-y_{2} f d z, d y_{2}-y_{3} f d z, d y_{3}\right) .
$$

As before $H$ is the subspace of $\Omega(K)^{3}$ consisting of the tuples $\left(\omega_{1}, \omega_{2}, \omega_{3}\right)$ for which there are formal local solutions at every point $p \in \mathbb{P}^{1}$. As before we consider the map $R: H \rightarrow H_{\mathrm{DR}}^{1}(X, \mathbb{C})$ that sends a tuple to $\overline{\omega_{3}}$, the image of $\omega_{3}$ in $H_{\mathrm{DR}}^{1}(X, \mathbb{C})$. We claim that $R$ is surjective.

Let $\omega_{3}$ with $\operatorname{Res}_{p}\left(\omega_{3}\right)=0$ be given. As in the proof of Proposition 8.15, there exists an $\omega_{2}$ such that the two equations $d y_{2}=y_{3} f d z+\omega_{2}$ and $d y_{3}=\omega_{3}$ have a formal local solution at each point of $\mathbb{P}^{1}$. The reason is that the residues of $\omega_{2}$ at the points of $S$ can be arbitrarily chosen, with the only restriction being that the sum of all residues is 0 . Now that $\omega_{2}$ is chosen, we want to produce an $\omega_{1}$ such that two equations $d y_{1}=y_{2} f d z+\omega_{1}$ and $d y_{2}=y_{3} f d z+\omega_{2}$ have a formal local 
solution at each point of $\mathbb{P}^{1}$. By construction, the last equation has a solution $y_{2}$, which is unique up to a constant. Using this constant one can prescribe the residues of $\omega_{1}$ at the points of $S$, with the only restriction being that the sum of all residues is 0 . This shows that $R$ is surjective.

Now $\lg l(L)$ is the direct sum of $H_{\mathrm{DR}}^{1}(X, \mathbb{C})$ and the cokernel of $L_{0}: K^{3} \rightarrow H_{0}$, where $H_{0} \subset H$ consists of the tuples in which $\omega_{3}$ is exact. After dividing by the subspace $\left\{L_{0}\left(0,0, y_{3}\right) \mid y_{3} \in K\right\}$, we have to calculate the cokernel of the map $L_{00}: K \oplus K \oplus \mathbb{C} \rightarrow H_{00}$ given by $\left(y_{1}, y_{2}, y_{3}\right) \mapsto\left(d y_{1}-y_{2} f d z, d y_{2}-y_{3} f d z, 0\right)$, where $H_{00} \subset H$ consists of the tuples with $\omega_{3}=0$.

Consider $\left(\omega_{1}, \omega_{2}, 0\right) \in H_{00}$. The equation $d y_{2}=y_{3} f d z+\omega_{2}$ is formally solvable at each point $p$ for a suitable $y_{3} \in \mathbb{C}$ (depending on $p$ ) if and only if $\operatorname{Res}_{p}\left(\omega_{2}\right)=0$ for every $p \notin S$. As in the proof of Lemma 8.4, this induces a linear map

$$
\begin{aligned}
R_{00}: H_{00} & \rightarrow\left\{\left(a_{p}\right)_{p \in S} \in \mathbb{C}^{S} \mid \sum a_{p}=0\right\} \\
\left(\omega_{1}, \omega_{2}, 0\right) & \mapsto\left(\operatorname{Res}_{p}\left(\omega_{2}\right)\right)_{p \in S} .
\end{aligned}
$$

The image of $f d z$ in the space is nonzero. We conclude that the dimension of the cokernel of $L_{00}$ is equal to $-2+\# S$ plus the dimension of the cokernel of $L_{000}: K \oplus K \oplus\{0\} \rightarrow H_{000}$, where $H_{000} \subset H_{00}$ consists of the tuples such that $\operatorname{Res}_{p}\left(\omega_{2}\right)=0$ for all $p$. By the case $n=2$, the cokernel has dimension $4 g-2+\# S$. Thus the cokernel of $L$ has dimension $(2 g-2+\# S)+(4 g-2+\# S)$.

\section{Regular singular differential equations}

Now we consider a differential operator $L$ on $\mathbb{P}^{1}$ which has only regular singularities. The aim is to calculate $\lg l(L)$. One can represent $L$ as a global connection $\nabla: \mathbb{C}(z)^{m} \rightarrow \Omega(\mathbb{C}(z) / \mathbb{C})^{m}$. Let Mer denote the sheaf of the meromorphic functions, and let $\Omega_{\text {mer }}$ denote the sheaf of the meromorphic differential forms on $\mathbb{P}^{1}$. One defines the sheaves $\mathscr{L}$ and $\mathscr{Q}$ on $\mathbb{P}^{1}$ by the exact sequence of sheaves

$$
0 \rightarrow \mathscr{L} \rightarrow \operatorname{Mer}^{m} \stackrel{\nabla}{\longrightarrow} \Omega_{\text {mer }}^{m} \stackrel{R}{\longrightarrow} 2 \rightarrow 0 .
$$

An element of $\operatorname{lgl}(L)$ is represented by an $\omega \in \Omega(\mathbb{C}(z) / \mathbb{C})^{m}$ having the property that the equation $\nabla(y)=\omega$ has of $\mathbb{P}^{1}$ a formal solution at every point $P$. The assumption that $L$ has only regular singular points implies that this formal solution is in fact meromorphic at the given point and therefore lies in the stalk $\operatorname{Mer}_{P}^{m}$, or equivalently $R(\omega)=0$.

First we analyze the sheaf 2. Let $t$ be a local parameter at $P$ (that is, $t=$ $z-c$ or $t=z^{-1}$ ). Locally at an open disk $D$ around $P$, the map $\nabla$ has the form $\nabla(y)=d y+A_{P}(y / t) d t$, where $A_{P}$ is a constant matrix such that the real part of each eigenvalue of $A_{P}$ lies in $[0,1)$. We note that $A_{P}=0$ if $P$ is not a singular point of $L$. For a singular point $P$ of $L$, we write $t_{0}(P)$ for the dimension of the 
kernel of $A_{P}$. We write $\operatorname{Res}_{P}: \Omega_{\text {mer,P }} \rightarrow \mathbb{C}_{P}^{m}$ for the residue map, and we write $\operatorname{Res}(\omega)=\sum_{P} \operatorname{Res}_{P}(\omega)[P]$. The latter is a section of the skyscraper sheaf $\bigoplus_{P} \mathbb{C}_{P}^{m}$ on $\mathbb{P}^{1}$.

An element $y \in \operatorname{Mer}_{P}^{m}$ can be written as $y=\sum_{n \gg-\infty} y_{n} t^{n}$ with all $y_{n} \in \mathbb{C}^{m}$, and an element $\omega \in \Omega_{\text {mer,P }}^{m}$ can be written as $\sum_{n \gg-\infty} \omega_{n} t^{n} d t$ with all $\omega_{n} \in \mathbb{C}^{m}$.

Now $\nabla(y)=\sum_{n \gg-\infty}\left(n+A_{P}\right) y_{n} t^{n-1} d t=\sum_{n \gg-\infty} \omega_{n} t^{n} d t$ has a solution if and only if $\omega_{-1}=\operatorname{Res}_{P}(\omega)$ lies in the image of $A_{P}$. It follows that 2 is the skyscraper sheaf $\bigoplus_{P}\left(\mathbb{C}_{P}^{m} / A_{P} \mathbb{C}_{P}^{m}\right)$ and $\mathscr{2}_{P}$ has dimension $t_{0}(P)$. Also $t_{0}(P)$ is the dimension of the space of solutions of $\nabla(y)=0$ locally at the point $P$.

The above exact sequence of sheaves is an acyclic resolution of $\mathscr{L}$ since the sheaves Mer and $\Omega_{\text {mer }}$ have trivial cohomology on every open subset of $\mathbb{P}^{1}$. Thus we can identify the cohomology groups of the complex

$$
0 \rightarrow \mathbb{C}(z)^{m} \stackrel{\nabla}{\longrightarrow} \mathbb{C}(z)^{m} d z \stackrel{R}{\longrightarrow} \bigoplus_{P} \mathscr{2}_{P} \rightarrow 0
$$

with $H^{*}\left(\mathbb{P}^{1}, \mathscr{L}\right)$. The definition of $\lg l(L)$ gives $\lg l(L) \cong H^{1}\left(\mathbb{P}^{1}, \mathscr{L}\right)$.

We will need a formula for the dimension of $H^{2}\left(\mathbb{P}^{1}, \mathscr{L}\right)$ (that is, the dimension of the cokernel of $R$ in the above complex). The image of Res : $\mathbb{C}(z)^{m} d z \rightarrow \bigoplus_{P} \mathbb{C}_{P}^{m}$ consists of the elements $v=\left(v_{P}\right)_{P}$ with $\sum v_{P}=0$. Let $p_{1}, \ldots, p_{r}$ denote the singular points of $L$, and let $A_{1}, \ldots, A_{r}$ be the corresponding constant matrices as above. Then one can verify that the cokernel of $R$ is isomorphic to $\mathbb{C}^{m} /\left(\sum_{i=1}^{r} A_{i}\left(\mathbb{C}^{m}\right)\right)$. Here we have identified all $\mathbb{C}_{P}^{m}$ with a single vector space $\mathbb{C}^{m}$. These identifications are not explicit, and we can only conclude that $\operatorname{dim} H^{2}\left(\mathbb{P}^{1}, \mathscr{L}\right)<m$ if $r>0$ and that $H^{2}\left(\mathbb{P}^{1}, \mathscr{L}\right)=0$ if some $A_{i}$ is invertible. The latter is equivalent to $t_{0}\left(p_{i}\right)=0$ and is again equivalent to the statement that the equation $\nabla(y)=0$ has no local solution $y \neq 0$ in a neighborhood of $p_{i}$.

Next, we will compute, for a singular point $p_{i}$ and a small disk $X_{i}$ around $p_{i}$, the dimensions of $H^{*}\left(X_{i}, \mathscr{L}\right)$ and $H^{*}\left(X_{i}^{*}, \mathscr{L}\right)$, where $X_{i}^{*}=X_{i} \backslash\left\{p_{i}\right\}$. Let $O_{\text {hol }}$ and $\Omega_{h o l}\left(\left[p_{i}\right]\right)$ denote the sheaves on $X_{i}$ of the holomorphic functions and the differential forms having at most a pole of order 1 at $p_{i}$. As before, the connection $\nabla: O_{\text {hol }}^{m} \rightarrow \Omega_{\text {hol }}\left(\left[p_{i}\right]\right)$ has the form $\nabla(y)=d y+A_{i}(y / t) d t$. Define the sheaf $\mathscr{P}$ by the exact sequence (above $X_{i}$ )

$$
0 \rightarrow \mathscr{L} \longrightarrow O_{\mathrm{hol}}^{m} \stackrel{\nabla}{\longrightarrow} \Omega_{\mathrm{hol}}\left(\left[p_{i}\right]\right)^{m} \longrightarrow \mathscr{P} \rightarrow 0 .
$$

Then $\mathscr{P}$ is a skyscraper sheaf with at most one nonzero stalk, namely, $\mathscr{P}_{p_{i}}=$ $\mathbb{C}^{m} / A_{i}\left(\mathbb{C}^{m}\right)$. The above sequence is an acyclic resolution of the restriction of $\mathscr{L}$ to $X_{i}$. Taking global sections on $X_{i}$ and $X_{i}^{*}$ one easily finds that the spaces $H^{0}\left(X_{i}, \mathscr{L}\right), H^{0}\left(X_{i}^{*}, \mathscr{L}\right)$ and $H^{1}\left(X_{i}^{*}, \mathscr{L}\right)$ have dimension $t_{0}\left(p_{i}\right)$ and that the other cohomology groups are 0 . 
Now we start a computation of $\chi\left(\mathbb{P}^{1}\right):=\sum(-1)^{i} \operatorname{dim} H^{i}\left(\mathbb{P}^{1}, \mathscr{L}\right)$. First write $X_{0}=\mathbb{P}^{1} \backslash\left\{p_{1}, \ldots, p_{r}\right\}$, and let $X_{i}$ denote, as before, a small disk around $p_{i}$ for $i=1, \ldots, r$. Further $X_{i}^{*}:=X_{i} \backslash\left\{p_{i}\right\}, \quad Y:=\bigcup_{i=1}^{r} X_{i}$ and $Y^{*}:=\bigcup_{i=1}^{r} X_{i}^{*}$. The Mayer-Vietoris exact sequence for the covering $X_{0} \cup Y$ of $\mathbb{P}^{1}$ reads

$$
\begin{aligned}
0 & \longrightarrow H^{0}\left(\mathbb{P}^{1}, \mathscr{L}\right) \longrightarrow H^{0}\left(X_{0}, \mathscr{L}\right) \oplus H^{0}(Y, \mathscr{L}) \longrightarrow H^{0}\left(Y^{*}, \mathscr{L}\right) \\
& \longrightarrow H^{1}\left(\mathbb{P}^{1}, \mathscr{L}\right) \longrightarrow H^{1}\left(X_{0}, \mathscr{L}\right) \oplus H^{1}(Y, \mathscr{L}) \longrightarrow H^{1}\left(Y^{*}, \mathscr{L}\right) \\
& \longrightarrow H^{2}\left(\mathbb{P}^{1}, \mathscr{L}\right) \longrightarrow H^{2}\left(X_{0}, \mathscr{L}\right) \oplus H^{2}(Y, \mathscr{L}) \longrightarrow H^{2}\left(Y^{*}, \mathscr{L}\right) \longrightarrow 0 .
\end{aligned}
$$

Let $\chi$ denote the Euler characteristic for the cohomology groups of $\mathscr{L}$ on the various open subsets. Then $\chi\left(\mathbb{P}^{1}\right)=\chi\left(X_{0}\right)+\chi(Y)-\chi\left(Y^{*}\right)$.

The restriction of $\mathscr{L}$ to $X_{0}$ is a locally constant sheaf of $\mathbb{C}$-vector spaces of dimension $m$. This corresponds to a representation of the fundamental group $\pi_{1}\left(X_{0}\right)$ on a vector space $\mathbb{C}^{m}$. The group $\pi_{1}\left(X_{0}\right)$ is free on $r-1$ generators $\gamma_{1}, \ldots, \gamma_{r-1}$. The cohomology groups that we want to calculate coincide with the group cohomology of the above representation. The latter are the cohomology groups of the complex $0 \rightarrow \mathbb{C}^{m} \rightarrow\left(\mathbb{C}^{m}\right)^{r-1} \rightarrow 0$, where the nontrivial map is given by $v \mapsto\left(\gamma_{1} v-v, \ldots, \gamma_{r-1} v-v\right)$. It follows that $\chi\left(X_{0}\right)=m-(r-1) m=m(2-r)$ and $H^{2}\left(X_{0}, \mathscr{L}\right)=0$. Using the local calculations we find the formula

$$
\chi\left(\mathbb{P}^{1}\right)=m(2-r)+\sum_{i=1}^{r} t_{0}\left(p_{i}\right) .
$$

Suppose that $t_{0}\left(p_{i}\right)=0$ for some $i$. Then $H^{i}\left(\mathbb{P}^{1}, \mathscr{L}\right)=0$ for $i=0,2$. Thus we proved the following result.

Proposition 9.1. Let $L$ be a connection on $\mathbb{P}^{1}$ of rank $m$ having $r>0$ regular singular points $p_{1}, \ldots, p_{r}$ (and no other singularities). Let $t_{0}\left(p_{i}\right)$ denote the dimension of the local solution space at the point $p_{i}$. Suppose that at least one $t_{0}\left(p_{i}\right)$ is zero. Then $\operatorname{lgl}(L)=(r-2) m-\sum_{i=1}^{r} t_{0}\left(p_{i}\right)$.

\section{References}

[Bertrand 1990] D. Bertrand, "Extensions de $D$-modules et groupes de Galois différentiels", pp. 125-141 in p-adic analysis (Trento, 1989), edited by F. Baldassarri et al., Lecture Notes in Math. 1454, Springer, Berlin, 1990. MR 92c:12006 Zbl 0732.13008

[Bertrand 1992] D. Bertrand, "Un analogue différentiel de la théorie de Kummer", pp. 39-49 in Approximations diophantiennes et nombres transcendants (Luminy, 1990), edited by P. Philippon, de Gruyter, Berlin, 1992. MR 93g:12007 Zbl 0785.12002

[Deligne 1970] P. Deligne, Équations différentielles à points singuliers réguliers, Lecture Notes in Math. 163, Springer, Berlin, 1970. MR 54 \#5232 Zbl 0244.14004

[Hardouin 2005] C. Hardouin, Structure galoisienne des extensions itérées de modules différentiels, Thesis, Université Paris VI, 2005, Available at http://www.iwr.uni-heidelberg.de/groups/compalg/ hardouin/thesclau2.pdf. 
[Van der Heiden 2005] G. J. van der Heiden, Weil pairing and the Drinfeld modular curve, Thesis, University of Groningen, 2005.

[Jacobson 1962] N. Jacobson, Lie algebras, Pure and Applied Mathematics 10, Wiley, New YorkLondon, 1962. MR 26\#1345 Zbl 0121.27504

[Jantzen 2003] J. C. Jantzen, Representations of algebraic groups, 2nd ed., Mathematical Surveys and Monographs 107, American Mathematical Society, Providence, RI, 2003. MR 2004h:20061 Zbl 1034.20041

[Katz 1990] N. M. Katz, Exponential sums and differential equations, Annals of Mathematics Studies 124, Princeton University Press, 1990. MR 93a:14009 Zbl 0731.14008

[Malgrange 1974] B. Malgrange, "Sur les points singuliers des équations différentielles", Enseignement Math. (2) 20 (1974), 147-176. MR 51 \#4316 Zbl 0299.34011

[Manin 1965] J. I. Manin, "Moduli fuchsiani”, Ann. Scuola Norm. Sup. Pisa (3) 19 (1965), 113-126. MR 31 \#4815 Zbl 0166.04301

[Van der Put and Reversat 2004] M. van der Put and M. Reversat, "Krichever modules for difference and differential equations", Astérisque 296 (2004), 207-225. MR 2006a:12005 Zbl 1086.12001

[Van der Put and Singer 2003] M. van der Put and M. F. Singer, Galois theory of linear differential equations, Grundlehren der Mathematischen Wissenschaften 328, Springer, Berlin, 2003. MR 2004c:12010 Zbl 1036.12008

[Varadarajan 1984] V. S. Varadarajan, Lie groups, Lie algebras, and their representations, Graduate Texts in Mathematics 102, Springer, New York, 1984. MR 85e:22001 Zbl 0955.22500

Received November 23, 2007. Revised April 20, 2008.

\author{
MARIUS VAN DER PUT \\ DEPARTMENT OF MATHEMATICS \\ UNIVERSITY OF GRONINGEN \\ P. O. BOX 407 \\ 9700 AK GRONINGEN \\ THE NETHERLANDS \\ mvdput@math.rug.nl \\ MARC REVERSAT \\ Institut DE MAThÉmatiques De Toulouse \\ C.N.R.S. U.M.R. 5219 \\ UNIVERSITÉ PAUL SABATIER \\ 118 ROUTE DE NARBONNE \\ 31062 TOULOUSE CEDEX 9 \\ FRANCE \\ marc.reversat@math.univ-toulouse.fr \\ http://www.math.univ-toulouse.fr/ reversat/
}

